Pacific

Journal of

Mathematics

\title{
VARIATIONS OF THE TELESCOPE CONJECTURE AND BOUSFIELD LATTICES FOR LOCALIZED CATEGORIES OF SPECTRA
}

F. LUKE WOLCOTT

Volume $276 \quad$ No. 2

August 2015 


\title{
VARIATIONS OF THE TELESCOPE CONJECTURE AND BOUSFIELD LATTICES FOR LOCALIZED CATEGORIES OF SPECTRA
}

\section{F. LUKE WOLCOTT}

\begin{abstract}
We investigate several versions of the telescope conjecture on localized categories of spectra and implications between them. Generalizing the "finite localization" construction, we show that, on such categories, localizing away from a set of strongly dualizable objects is smashing. We classify all smashing localizations on the harmonic category, $H \mathbb{F}_{p}$-local category and $I$-local category, where $I$ is the Brown-Comenetz dual of the sphere spectrum; all are localizations away from strongly dualizable objects, although these categories have no nonzero compact objects. The Bousfield lattices of the harmonic, $E(n)$-local, $K(n)$-local, $H \mathbb{F}_{p}$-local and $I$-local categories are described, along with some lattice maps between them. One consequence is that in none of these categories is there a nonzero object that squares to zero. Another is that the $\boldsymbol{H} \mathbb{F}_{p}$-local category has localizing subcategories that are not Bousfield classes.
\end{abstract}

\section{Introduction}

The telescope conjecture, first stated by Ravenel [1984, Conjecture 10.5], is a claim about two classes of localization functors in the $p$-local stable homotopy category of spectra. First, one can localize away from a finite type $n+1$ spectrum $F(n+1)$; the acyclics are the smallest localizing subcategory containing $F(n+1)$, and we denote this functor by $L_{n}^{f}$. Second, one can localize at the wedge of the first $n+1$ Morava $K$-theories $K(0) \vee \cdots \vee K(n)$; the acyclics are all spectra that smash with $K(0) \vee \cdots \vee K(n)$ to zero and this is denoted $L_{n}$. Both these localizations are smashing, i.e., they commute with coproducts. The telescope conjecture $\left(\mathrm{TC}_{n}\right)$ claims that $L_{n}^{f}$ and $L_{n}$ are isomorphic. In fact, here we consider three slightly different versions, $\mathrm{TC} 1_{n}, \mathrm{TC} 2_{n}$, and $\mathrm{TC} 3_{n}$, of the telescope conjecture. In Section 3 we articulate them carefully and show implications between them.

The conjecture is known to hold for $n=0$ [Ravenel 1992, p. 79] and for $n=1$ when $p=2$ [Mahowald 1982] and $p>2$ [Miller 1981]. A valiant but unsuccessful

MSC2010: 55P42, 18E30, 55U35.

Keywords: telescope conjecture, Bousfield localization, Bousfield lattice, harmonic category, smashing localization. 
effort at a counterexample, for $n \geq 2$, was undertaken by Mahowald, Ravenel, and Shick, as outlined in [Mahowald et al. 2001]. Since then little progress has been made, and the original conjecture remains open.

A generalization of the telescope conjecture can be stated for spectra, as well as other triangulated categories. Localization away from a finite spectrum, i.e, a compact object of the category, always yields a smashing localization functor (see, e.g., [Bousfield 1979a, Proposition 2.9] or [Miller 1992] or [Hovey et al. 1997, Theorem 3.3.3]). The Generalized Smashing Conjecture (GSC) is that every smashing localization arises in this way. If true, then every smashing localization is determined by its compact acyclics; if the GSC holds in spectra, then so must the $\mathrm{TC}_{n}$ for all $n$.

The GSC, essentially stated for spectra decades ago by Bousfield [1979b, Conjecture 3.4], has been formulated in many other triangulated categories, in many cases labeled as the telescope conjecture, and in many cases proven to hold. Neeman [1992] made the conjecture for the derived category $D(R)$ of a commutative ring $R$ and showed that it holds when the ring is Noetherian. See also [Hovey et al. 1997, Theorem 6.3.7] or [Krause and Št'ovíček 2010] for a generalization. On the other hand, Keller [1994] gave an example of a non-Noetherian ring for which the GSC fails. Benson, Iyengar, and Krause have shown that the GSC holds in a stratified category [Benson et al. 2011a], such as the stable module category of a finite group [Benson et al. 2011b]. Balmer and Favi [2011] showed that in a tensor triangulated category with a good notion of support, the GSC is a "local" question.

It is worth noting that there are further variations of the GSC that we will not consider here. Krause [2000] formulated a variation of the GSC, in terms of subcategories generated by sets of maps, that makes sense (and holds) for any compactly generated triangulated category. Krause and Solberg [2003] gave a variation for stable module categories, stated in terms of cotorsion pairs. See also [Krause 2005; Angeleri Hügel et al. 2008; Brüning 2007; Št'ovíček 2010].

To date, Keller's ring yields the only category where the GSC is known to fail. In this paper we give several more examples. Incidentally, each is a well generated triangulated category that is not compactly generated.

One of our main results is the following. We weaken the assumptions for "finite localization" and show that, in many categories, localization away from any set of strongly dualizable objects yields a smashing localization. (Recall that an object $X$ is strongly dualizable if $F(X, Y) \cong F(X, \mathbb{1}) \wedge Y$ for all $Y$, where $\mathbb{1}$ is the tensor unit and $F(-,-)$ the function object bifunctor.) Let $\operatorname{loc}(X)$ denote the smallest localizing subcategory containing $X$. We prove the following as Theorem 3.5.

Theorem (A). Let $\mathrm{T}$ be a well generated tensor triangulated category such that $\operatorname{loc}(\mathbb{1})=\mathrm{T}$. Let $A=\left\{B_{\alpha}\right\}$ be a (possibly infinite) set of strongly dualizable objects. Then there exists a smashing localization functor $L: \mathrm{T} \rightarrow \mathrm{T}$ with $\operatorname{Ker} L=\operatorname{loc}(A)$. 
Thus we are led to conjecture the following.

Conjecture (Strongly Dualizable Generalized Smashing Conjecture (SDGSC)). Every smashing localization is localization away from a set of strongly dualizable objects.

We give several examples of categories where the GSC fails but the SDGSC holds. In fact, we consider a topological setting, where one can also formulate a version (or versions, rather) of the original telescope conjecture.

Specifically, we consider localized categories of spectra. Let $\mathcal{S}$ be the $p$-local stable homotopy category, and let $\wedge$ denote the smash (i.e., tensor) product. Take $Z$ to be an object of $\mathcal{S}$, and let $L=L_{Z}: \mathcal{S} \rightarrow \mathcal{S}$ be the localization functor that annihilates $Z_{*}$-acyclic objects. The full subcategory of $L$-local objects, that is, objects $X$ for which $X \rightarrow L X$ is an equivalence, has a tensor triangulated structure induced by that of $\mathcal{S}$. Let $\mathcal{L}$ denote this category; the triangles are the same as in $\mathcal{S}$, the coproduct is $X \coprod_{\mathcal{L}} Y=L(X \bigsqcup Y)$ and the tensor is $X \wedge_{\mathcal{L}} Y=L(X \wedge Y)$.

In Definition 3.6, we define localization functors $l_{n}^{f}$ and $l_{n}$ on $\mathcal{L}$ that are localized versions of $L_{n}^{f}$ and $L_{n}$. The localized telescope conjecture (LTC) claims that $l_{n}^{f}$ and $l_{n}$ are isomorphic. In fact, we give three versions of the LTC and in Theorems 3.12 and 3.13 establish implications between them. Then, examining specific examples of localized categories of spectra, we conclude the following in Theorems 4.3, 5.11, 6.1, 6.5 and 6.9 and Corollary 5.6.

Theorem (B). All versions of the localized telescope conjecture, $\mathrm{LTC}_{i}, \mathrm{LTC}_{i}$, and $\mathrm{LTC}_{i}$ hold for all $i \geq 0$, in the $\bigvee_{n \geq 0} K(n)$-local (i.e., harmonic), $K(n)$-local, $H \mathbb{F}_{p}$-local, BP-local, and I-local categories, where I is the Brown-Comenetz dual of the sphere spectrum.

In order to consider the GSC and SDGSC in $\mathcal{L}$, we must classify the smashing localizations on $\mathcal{L}$. We are able to do this in several examples.

Theorem (C). In the harmonic category, the GSC fails but the SDGSC holds, and likewise in the $H \mathbb{F}_{p}$-local and I-local categories. In the BP-local category the GSC fails but the SDGSC is open. In the E(n)-local and $K(n)$-local categories the GSC and SDGSC both hold.

Proof. This theorem is concluded from Theorems 4.4 and 5.11, Propositions 6.3 and 6.10, and Corollaries 5.6 and 6.7.

One novelty in our approach is our use of Bousfield lattice arguments. Given an object $X$ in a tensor triangulated category T, the Bousfield class of $X$ is defined by $\langle X\rangle=\{W \mid W \wedge X=0\}$. It is now known [Iyengar and Krause 2013] that every well generated tensor triangulated category has a set of Bousfield classes. This set has the structure of a lattice and is called the Bousfield lattice of $T$. One can now attempt to calculate the Bousfield lattices of categories of localized spectra. Furthermore, every 
smashing localization yields a pair of so-called complemented Bousfield classes. Information about the Bousfield lattice of a category gives information about its complemented classes, which gives information about the smashing localization functors on the category.

Moreover, the first version of the telescope conjecture $\mathrm{TC}_{n}$ is that two spectra $T(n)$ and $K(n)$ have the same Bousfield class. In the localized version this becomes $\left(\operatorname{LTC}_{n}\right)$ the claim that $\langle L T(n)\rangle=\langle L K(n)\rangle$ in the Bousfield lattice of $\mathcal{L}$. One is thus led to investigating Bousfield lattices of localized spectra.

Corollary 2.7 gives an upper bound, $2^{2^{\mathrm{N}_{0}}}$, on the cardinality of such lattices. Jon Beardsley has calculated the Bousfield lattice of the harmonic category to be isomorphic to the power set of $\mathbb{N}$; we give this calculation in Proposition 4.2. In Corollary 5.4 and Proposition 5.7 we show that one can realize this lattice as an inverse limit of the Bousfield lattices of $E(n)$-local categories, as $n$ ranges over $\mathbb{N}$. Then in Corollary 5.10 and Propositions 6.2 and 6.6, we show that the $K(n)$-local, $H \mathbb{F}_{p}$-local, and $I$-local categories all have two-element Bousfield lattices. In Proposition 6.11 we give a lower bound, $2^{\aleph_{0}}$, on the cardinality of the Bousfield lattice of the $B P$-local category.

One immediate object-level application of these Bousfield lattice calculations is the following. Call an object $X$ square-zero if it is nonzero but $X \wedge X=0$. Then Proposition 2.9 shows that there are no square-zero objects in the harmonic, $E(n)$-, $K(n)$-, $H \mathbb{F}_{p^{-}}$, or $I$-local categories.

We are also able to answer the analogue of a conjecture by Hovey and Palmieri, originally stated for the stable homotopy category. Conjecture 9.1 in [Hovey and Palmieri 1999] is that every localizing subcategory is a Bousfield lattice. Proposition 6.4 demonstrates that this fails in the $H \mathbb{F}_{p}$-local category by giving two localizing subcategories that are not Bousfield classes.

Section 2 establishes the categorical setting, and provides background on localization, Bousfield lattices, and stable homotopy theory. Section 3 defines the various versions of the telescope conjecture, for spectra and for localized spectra, and establishes implications among them. The remainder of the paper is devoted to examining specific examples: the harmonic category (Section 4), the $E(n)$-local and $K(n)$-local categories (Section 5), and the $H \mathbb{F}_{p}$-local, $I$-local, $B P$-local, and $F(n)$-local categories (Section 6). All results are new unless cited. Most of the results on the $E(n)$-local and $K(n)$-local categories in Section 5 follow in a straightforward way from Hovey and Strickland's work [1999] and are included for completeness.

\section{Preliminaries}

2A. Categorical setting. We start with the notion of a tensor triangulated category C, i.e., a triangulated category with set-indexed coproducts and a closed 
symmetric monoidal structure compatible with the triangulation [Hovey et al. 1997, Appendix A]. Let $\Sigma: \mathrm{C} \rightarrow \mathrm{C}$ denote the shift and $[X, Y]$ the morphisms from $X$ to $Y$, and let $[X, Y]_{n}=\left[\Sigma^{n} X, Y\right]$ for any $n \in \mathbb{Z}$.

Let $-\wedge-$ denote the smash (tensor) product, $\mathbb{1}$ the unit, and $F(-,-)$ the function object bifunctor; $F(X,-)$ is the right adjoint to $X \wedge-$. Recall that an object $X$ in $C$ is said to be strongly dualizable if the natural map $D X \wedge Y \rightarrow F(X, Y)$ is an isomorphism for all $Y$, where $D X=F(X, \mathbb{1})$ is the Spanier-Whitehead dual. Since $F(\mathbb{1}, X) \cong X$ for all $X$, the map $F(\mathbb{1}, \mathbb{1}) \wedge Y \rightarrow F(\mathbb{1}, Y)$ is an equivalence and $\mathbb{1}$ is always strongly dualizable.

For a regular cardinal $\alpha$, we say an object $X$ is $\alpha$-small if every morphism $X \rightarrow \bigsqcup_{i \in I} Y_{i}$ factors through $\bigsqcup_{i \in J} Y_{i}$ for some $J \subseteq I$ with $|J|<\alpha$. If $X$ is $\aleph_{0}$-small we say $X$ is compact ([Hovey et al. 1997] calls this small); this is equivalent to the condition that the natural map $\bigoplus_{i \in K}\left[X, Z_{i}\right] \rightarrow\left[X, \bigsqcup_{i \in K} Z_{i}\right]$ is an isomorphism for any set-indexed coproduct $\bigsqcup_{i \in K} Z_{i}$. We say $C$ is $\alpha$-well generated if it has a set of perfect generators [Krause 2010, Section 5.1] which are $\alpha$-small, and C is well generated if it is $\alpha$-well generated for some $\alpha$. See [Krause 2010] for more details.

A localizing subcategory is a triangulated subcategory of $C$ that is closed under retracts and coproducts; a thick subcategory is a triangulated subcategory that is closed under retracts. Given an object or set of objects $X$, let loc $(X)(\operatorname{resp} . \operatorname{th}(X))$ denote the smallest localizing (resp. thick) subcategory containing $X$. We say that $\operatorname{loc}(X)$ is generated by $X$.

Notation 2.1. Throughout this paper let $T$ be a well generated tensor triangulated category such that $\operatorname{loc}(\mathbb{1})=\mathrm{T}$.

In the language of [Hovey et al. 1997], such a T is almost a "monogenic stable homotopy category", except that we do not insist that the unit $\mathbb{1}$ is compact.

In practice, in this paper $T$ will always be either the $p$-local stable homotopy category of spectra $\mathcal{S}$ or the category $\mathcal{L}_{Z}$ of $L_{Z}$-local objects derived from a localization functor $L_{Z}: \mathcal{S} \rightarrow \mathcal{S}$. The former satisfies Notation 2.1 by [Hovey et al. 1997, Example 1.2.3(a)], and the latter by Theorem 2.3 and Lemma 2.4 below.

2B. Background on localization. Recall that a localization functor (or simply localization) on a tensor triangulated category $\mathrm{C}$ is an exact functor $L: \mathrm{C} \rightarrow \mathrm{C}$, along with a natural transformation $\eta: 1 \rightarrow L$ such that $L \eta$ is an equivalence and $L \eta=\eta L$. We call $\operatorname{Ker} L$ the $L$-acyclics. It follows that there is an exact functor $C: \mathrm{C} \rightarrow \mathrm{C}$, called colocalization, such that every $X$ in $\mathrm{C}$ fits into an exact triangle $C X \rightarrow X \rightarrow L X$, with $C X L$-acyclic. An object $Y$ is $L$-local if it is in the essential image of $L$, and this is equivalent to satisfying $[Z, Y]=0$ for all $L$-acyclic $Z$. See [Hovey et al. 1997, Chapter 3] or [Krause 2010] for further background.

We also recall two special types of localizations. A localization $L: \mathrm{C} \rightarrow \mathrm{C}$ is said to be smashing if $L$ preserves coproducts, equivalently if $L X \cong L \mathbb{1} \wedge X$ for all $X$. 
Given a set $A$ of objects of $\mathrm{C}$, we say that a localization functor $L: \mathrm{C} \rightarrow \mathrm{C}$ is localization away from $A$ if the $L$-acyclics are precisely $\operatorname{loc}(A)$. If such a localization exists, we also say it is generated by $A$. When $C=\mathcal{S}$, it is well known (e.g., [Miller 1992; Mahowald and Sadofsky 1995]) that localization away from a set of compact objects exists and yields a smashing localization functor. As mentioned in the introduction, this result has been generalized to other categories as well (e.g., [Hovey et al. 1997, Theorem 3.3.3], [Balmer and Favi 2011, Theorem 4.1]). We present a further generalization in Theorem 3.5.

In this paper we will restrict our attention to homological localizations, which we now describe. Given an object $Z$ in a tensor triangulated category $C$, the Bousfield class of $Z$ is defined to be

$$
\langle Z\rangle=\{W \in C \mid W \wedge Z=0\} .
$$

Extending a classical result of Bousfield's for $\mathcal{S}$, Iyengar and Krause recently showed [2013, Proposition 2.1] that for every object $Z$ in a well generated tensor triangulated category $\mathrm{C}$, there is a localization functor $L_{Z}: \mathrm{C} \rightarrow \mathrm{C}$ with $L_{Z}$-acyclics precisely $\langle Z\rangle$. We call such an $L_{Z}$ a homological localization at $Z$.

Notation 2.2. Let $T$ be as in Notation 2.1, with tensor unit $\mathbb{1}$. For an object $Z$ in $T$, let $L_{Z}: \mathrm{T} \rightarrow \mathrm{T}$ be homological localization at $Z$. Let $\mathcal{L}_{Z}$ denote the category of $L_{Z}$-local objects, the essential image of $L_{Z}$.

Theorem 2.3 [Hovey et al. 1997, 3.5.1, 3.5.2]. Let $L=L_{Z}: T \rightarrow T$ be a localization, and $\mathcal{L}_{Z}$ the category of $L_{Z}$-local objects. Then $\mathcal{L}_{Z}$ has a natural structure as a tensor triangulated category, generated by $L_{Z} \mathbb{1}$, which is the unit. Considered as a functor from $\mathrm{T}$ to $\mathcal{L}_{Z}, L$ preserves triangles, the tensor product and its unit, coproducts, and strong dualizability. Furthermore, L preserves compactness if and only if $L$ is a smashing localization.

Explicitly, for $L$-local objects $X, X_{i}$ and $Y$, in $\mathcal{L}$ we have $\bigsqcup_{\mathcal{L}} X_{i}=L\left(\bigsqcup_{\mathrm{\top}} X_{i}\right)$ and $X \wedge_{\mathcal{L}} Y=L\left(X \wedge_{\mathrm{T}} Y\right)$ and $F_{\mathcal{L}}(X, Y)=F(X, Y)$. Note that $L_{Z} \mathbb{1}$ is strongly dualizable but may not be compact in $\mathcal{L}_{Z}$.

Lemma 2.4. The category $\mathcal{L}_{Z}$ is well generated.

Proof. By Proposition 2.1 of [Iyengar and Krause 2013], the $L_{Z}$-acyclics $\langle Z\rangle$ form a well generated localizing subcategory of $T$. Then by [Krause 2010, Theorem 7.2.1], the Verdier quotient $\mathrm{T} /\langle Z\rangle$, which is equivalent to the local category $\mathcal{L}_{Z}$, is well generated.

We conclude this subsection with a lemma containing four useful well-known facts. Recall that a ring object in a tensor triangulated category is an object $R$ with an associative multiplication map $\mu: R \wedge R \rightarrow R$ and a unit $\iota: \mathbb{1} \rightarrow R$, making the evident diagrams commute. If $R$ is a ring object, then an $R$-module object is an 
object $M$ with a map $m: R \wedge M \rightarrow M$ along with evident commutative diagrams. Note that $R \wedge X$ is an $R$-module object for every $X$. A skew field object is a ring object such that every $R$-module object is free, i.e., isomorphic to a coproduct of suspensions of $R$ [Hovey et al. 1997, Definition 3.7.1].

Lemma 2.5. Let $\mathrm{C}$ be a tensor triangulated category with $\operatorname{loc}(\mathbb{1})=\mathrm{C}$, and $L: \mathrm{C} \rightarrow \mathrm{C}$ a localization.

(1) Every localizing subcategory $\mathrm{S}$ of $\mathrm{C}$ is tensor-closed; that is, if $X \in \mathrm{S}$ and $Y \in \mathrm{C}$, then $X \wedge Y \in \mathrm{S}$.

(2) For all $X$ and $Y$ in $C, L(X \wedge Y)=L(L X \wedge L Y)$.

(3) Considered as a functor from $\mathrm{C}$ to $\mathcal{L}, L$ also preserves ring objects and module objects.

(4) If $R$ is a ring object and $M$ is an $R$-module object (in particular, if $M=R$ ), then $M$ is $R$-local.

Proof. For (1), note that $Y \in \operatorname{loc}(\mathbb{1})=\mathrm{C}$, so $X \wedge Y \in \operatorname{loc}(X \wedge \mathbb{1})=\operatorname{loc}(X) \subseteq \mathrm{S}$.

For (2), consider the exact triangle $X \wedge C Y \rightarrow X \wedge Y \rightarrow X \wedge L Y$. Since $C Y$ is $L$-acyclic and these form a localizing subcategory, $L(X \wedge C Y)=0$, so $L(X \wedge Y)=L(X \wedge L Y)$. Using the same reasoning with the triangle $C X \wedge L Y \rightarrow$ $X \wedge L Y \rightarrow L X \wedge L Y$, the result follows.

If $R \in \mathrm{C}$ is a ring object, then $L(\mu): L(R \wedge R)=L(L R \wedge L R)=L R \wedge_{\mathcal{L}} L R \rightarrow L R$, and all the localized diagrams commute. A similar argument applies to module objects, completing part (3).

Part (4) is [Ravenel 1984, Proposition 1.17(a)].

2C. Background on Bousfield lattices. Every well generated tensor triangulated category, and hence every localized category of spectra, has a set (rather than a proper class) of Bousfield classes [Iyengar and Krause 2013, Theorem 3.1]. This was also recently shown for every tensor triangulated category with a combinatorial model [Casacuberta et al. 2014]. This set is called the Bousfield lattice BL(T) and has a lattice structure which we now recall. Refer to [Hovey and Palmieri 1999; Wolcott 2014] for more details.

The partial ordering is given by reverse inclusion: we say $\langle X\rangle \leq\langle Y\rangle$ when $W \wedge Y=0$ implies $W \wedge X=0$. It is also helpful to remember that, unwinding definitions, $\langle X\rangle \leq\langle Y\rangle$ precisely when every $L_{X}$-local object is also $L_{Y}$-local. Clearly $\langle 0\rangle$ is the minimum and $\langle\mathbb{1}\rangle$ is the maximum class. The join of any set of classes is $\bigvee_{i \in I}\left\langle X_{i}\right\rangle=\left\langle\bigsqcup_{i \in I} X_{i}\right\rangle$, and the meet is defined to be the join of all lower bounds.

The smash product induces an operation on Bousfield classes, where $\langle X\rangle \wedge\langle Y\rangle$ is equal to $\langle X \wedge Y\rangle$. This is a lower bound, but in general not the meet. However, if we restrict to the subposet $\mathrm{DL}=\{\langle W\rangle \mid\langle W \wedge W\rangle=\langle W\rangle\}$, then the meet and smash 
agree. Since coproducts distribute across the smash product, DL is a distributive lattice.

We say a class $\langle X\rangle$ is complemented if there exists a class $\left\langle X^{c}\right\rangle$ such that $\langle X\rangle \wedge\left\langle X^{c}\right\rangle=\langle 0\rangle$ and $\langle X\rangle \vee\left\langle X^{c}\right\rangle=\langle\mathbb{1}\rangle$. The collection of complemented classes is denoted BA. For example, every smashing localization $L: T \rightarrow T$ gives a pair of complemented classes, namely $\langle C \mathbb{1}\rangle$ and $\langle L \mathbb{1}\rangle$. Because every complemented class is also in DL, BA is a Boolean algebra.

Proposition 2.6. Let $\mathrm{T}$ be as in Notation 2.1, and $L_{Z}: \mathrm{T} \rightarrow \mathrm{T}$ a localization functor as in Notation 2.2. Then $L_{Z}$ induces a well-defined order-preserving map of lattices $\mathrm{BL}(\mathrm{T}) \rightarrow \mathrm{BL}\left(\mathcal{L}_{Z}\right)$, where $\langle X\rangle \mapsto\left\langle L_{Z} X\right\rangle$. This map is surjective and sends $\mathrm{DL}(\mathrm{T})$ onto $\mathrm{DL}\left(\mathcal{L}_{Z}\right)$ and $\mathrm{BA}(\mathrm{T})$ onto $\mathrm{BA}\left(\mathcal{L}_{Z}\right)$.

Proof. Most of this is proved in Lemma 3.1 of [Wolcott 2014]. For $\langle X\rangle \in \mathrm{DL}(\mathrm{T})$, using Lemma 3.10 we get

$$
\langle L X\rangle=\langle L(X \wedge X)\rangle=\langle L(L X \wedge L X)\rangle=\left\langle L X \wedge_{\mathcal{L}} L X\right\rangle .
$$

Likewise, one can check that for $\langle X\rangle \in \mathrm{BA}(\mathrm{T})$, the class $\langle L X\rangle \in \mathrm{BL}(\mathcal{L})$ is complemented by $\left\langle L\left(X^{c}\right)\right\rangle$, keeping in mind that $\langle L \mathbb{1}\rangle$ is the top class in $\operatorname{BL}(\mathcal{L})$.

Corollary 2.7. For any $Z \in \mathcal{S}$, we have $\left|\mathrm{BL}\left(\mathcal{L}_{Z}\right)\right| \leq 2^{2^{\mathrm{N}_{0}}}$.

Proof. Observe that $\left|\mathrm{BL}\left(\mathcal{L}_{Z}\right)\right| \leq|\mathrm{BL}(\mathcal{S})| \leq 2^{2^{\aleph_{0}}}$, where the second inequality is proved in [Ohkawa 1989].

Lemma 2.8. Let $\mathrm{T}$ be as in Notation 2.1, and $X$ and $Y$ objects of $\mathrm{T}$. Then $\langle X\rangle \leq\langle Y\rangle$ if and only if $L_{X}=L_{X} L_{Y}=L_{Y} L_{X}$ and in this case the following diagram commutes (also with $\mathrm{BL}$ replaced by $\mathrm{DL}$ or $\mathrm{BA}$ ).

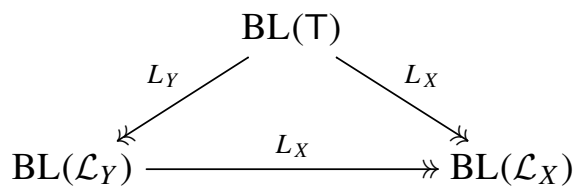

Proof. The first equivalence is straightforward; it follows from [Ravenel 1984, Proposition 1.22] and the observation that $\langle X\rangle \leq\langle Y\rangle$ precisely when all $L_{X}$-locals are $L_{Y}$-locals. The last remark follows from Proposition 2.6.

Here we mention one object-level application of the Bousfield lattice calculations of Sections 5 and 6.

Proposition 2.9. There are no square-zero objects in the harmonic, $E(n)-, K(n)-$, $H \mathbb{F}_{p^{-}}$, or I-local categories.

Proof. In Corollary 2.8 of [Wolcott 2014], we show that in a well generated tensor triangulated category, there are no square-zero objects if and only if $\mathrm{BA}=\mathrm{DL}=\mathrm{BL}$. The claim follows from Corollaries 5.4 and 5.10 and Propositions 6.2 and 6.6. 
2D. Background on spectra. We quickly review some relevant background on the stable homotopy category. See [Ravenel 1992; 1993; Hovey 1995a; Mahowald and Sadofsky 1995] for more details. Fix a prime $p$ and let $\mathcal{S}$ denote the $p$-local stable homotopy category of spectra. Let $S^{0}$ denote the sphere spectrum. The finite spectra $\mathcal{F}$ are the compact objects of $\mathcal{S}$, and $\mathcal{F}=\operatorname{th}\left(S^{0}\right)$. The structure of $\mathcal{F}$ is determined by the Morava $K$-theories $K(i)$. For each $i \geq 0, K(i)$ is a skew field object in $\mathcal{S}$, such that $K(i) \wedge K(j)=0$ when $i \neq j$. If $X$ is a finite spectrum and $K(j) \wedge X=0$, then $K(j-1) \wedge X=0$. We say a finite spectrum $X$ is type $n$ if $n$ is the smallest integer such that $K(n) \wedge X \neq 0$. Define $\mathcal{C}_{n}=\langle K(n-1)\rangle \cap \mathcal{F}$. Then every thick subcategory of $\mathcal{F}$ is $\mathcal{C}_{n}$ for some $n$. It follows that any two spectra of type $n$ generate the same thick subcategory, and hence Bousfield class; let $F(n)$ denote a generic type $n$ spectrum.

Given a type $n$ spectrum $X$, there is a $v_{n}$ self-map $f: \Sigma^{d} X \rightarrow X$ for which $\left[S^{0}, K(n) \wedge f\right]_{i}$ is an isomorphism for all $i$ and for which $\left[S^{0}, K(m) \wedge f\right]_{j}=0$ for all $j$ and $m \neq n$. We define $f^{-1} X$ to be the telescope, i.e., sequential or homotopy colimit, of the diagram $X \rightarrow \Sigma^{-d} X \rightarrow \cdots$. By the periodicity theorem, any choice of $v_{n}$ self-map $f$ yields an isomorphic telescope. The telescopes of different type $n$ spectra are Bousfield equivalent; denote this class by $\langle T(n)\rangle$.

As mentioned above, localization away from a finite spectrum $F(n+1)$ exists and is smashing. This localization functor is denoted $L_{n}^{f}$ and is the same as homological localization at $T(0) \vee \cdots \vee T(n)$.

Let $E(n)$ denote the Johnson-Wilson spectrum; this is a ring spectrum with $\langle E(n)\rangle=\langle K(0) \vee \cdots \vee K(n)\rangle$. Define $L_{n}: \mathcal{S} \rightarrow \mathcal{S}$ to be homological localization at $E(n)$. A deep theorem of Ravenel [1992, Theorem 7.5.6] shows that $L_{n}$ is smashing for all $n$. The functors $L_{n}^{f}$ and $L_{n}$ are the only known smashing localization functors on $\mathcal{S}$.

The $L_{n}^{f}$-acyclics are given by $\operatorname{loc}(F(n+1))=\operatorname{loc}\left(\mathcal{C}_{n+1}\right)=\operatorname{loc}(\langle K(n)\rangle \cap \mathcal{F})=$ $\operatorname{loc}(\langle E(n)\rangle \cap \mathcal{F})$. The $L_{n}$-acyclics are $\langle E(n)\rangle=\operatorname{loc}(\langle E(n)\rangle)$. Thus every $L_{n}^{f}$-acyclic is $L_{n}$-acyclic, and we have $\langle K(0) \vee \cdots \vee K(n)\rangle \leq\langle T(0) \vee \cdots \vee T(n)\rangle$ for all $n$. It follows that there is a natural map $L_{n}^{f} \rightarrow L_{n}$.

For convenience later, we collect some calculations in $\mathcal{S}$.

Lemma 2.10. In $\mathrm{BL}(\mathcal{S})$ we have the following.

(1) $\langle F(m)\rangle \leq\langle F(n)\rangle$ if and only if $m \geq n$. For all $n$ and $m,\langle F(m) \wedge F(n)\rangle \neq\langle 0\rangle$. Furthermore, $\langle F(n) \wedge F(n)\rangle=\langle F(n)\rangle$ for all $n$.

(2) $\langle F(m) \wedge T(n)\rangle=\langle 0\rangle$ when $m>n$, and $\langle F(m) \wedge T(n)\rangle=\langle T(n)\rangle$ when $m \leq n$.

(3) $\langle T(m) \wedge T(n)\rangle=\langle 0\rangle$ when $m \neq n$, and $\langle T(n) \wedge T(n)\rangle=\langle T(n)\rangle$.

(4) $\langle F(m) \wedge K(n)\rangle=\langle 0\rangle$ when $m>n$, and $\langle K(n)\rangle=\langle F(m) \wedge K(n)\rangle \leq\langle F(m)\rangle$ when $m \leq n$. 
(5) $\langle T(m) \wedge K(n)\rangle=\langle 0\rangle$ when $m \neq n$, and $\langle K(n)\rangle=\langle T(n) \wedge K(n)\rangle \leq\langle T(n)\rangle$.

(6) $\langle K(m) \wedge K(n)\rangle=\langle 0\rangle$ when $m \neq n$, and $\langle K(n) \wedge K(n)\rangle=\langle K(n)\rangle$.

Proof. Part (1) is Theorem 14 of [Hopkins and Smith 1998], along with the observation that $\langle F(n)\rangle$ is complemented by $\left\langle L_{n-1}^{f} S^{0}\right\rangle$, and hence is in DL.

Part (2) is in [Ravenel 1993, 2.8(i)], [Mahowald and Sadofsky 1995, 6.2], and [Hovey and Palmieri 1999, Section 5]. Part (3) is also in [Hovey and Palmieri 1999, Section 5].

Part (4) follows from the definition of type $m$ spectra. Since each $K(i)$ is a skew field object, $F(m) \wedge K(i) \neq\langle 0\rangle$ implies this $K(i)$-module object $F(m) \wedge K(i)$ is a wedge of suspensions of $K(i)$.

From the periodicity theorem, $T(m)$ has nonzero $K(m)$ homology, and therefore $T(m) \wedge K(m) \neq 0$. The rest of Part (5) is in [Ravenel 1992, Proposition A.2.13]. Finally, Part (6) is well known.

Let $I$ denote the Brown-Comenetz dual of the sphere spectrum. Recall that a harmonic spectrum is one that is local with respect to $\bigvee_{i \geq 0} K(i)$. The following theorem plays a large role in the results in this paper.

Theorem 2.11. There are no nonzero compact objects in the following categories.

(1) The BP-local category

(2) The harmonic category

(3) The HZZ-local category

(4) The $H \mathbb{F}_{p}$-local category

(5) The I-local category

Proof. This is Corollary B.13 in [Hovey and Strickland 1999].

\section{Local versions of the telescope conjecture}

In this section, let $L=L_{Z}: \mathcal{S} \rightarrow \mathcal{S}$ be a localization functor for some $Z \in \mathcal{S}$, and let $\mathcal{L}=\mathcal{L}_{Z}$ denote the category of $L$-locals. First we state the various versions of the original telescope conjecture on $\mathcal{S}$.

Definition 3.1. Fix an integer $n \geq 0$. On $\mathcal{S}$, we have the following versions of the telescope conjecture.

$\mathrm{TC}_{n} \quad\langle T(n)\rangle=\langle K(n)\rangle$.

$\mathrm{TC} 2_{n} \quad L_{n}^{f} X \stackrel{\sim}{\rightarrow} L_{n} X$ for all $X$.

$\mathrm{TC}_{n} \quad$ If $X$ is type $n$ and $f$ is a $v_{n}$ self-map, then $L_{n} X \cong f^{-1} X$.

GSC Every smashing localization is generated by a set of compact objects.

SDGSC Every smashing localization is generated by a set of strongly dualizable objects. 
Theorem 3.2. On the category $\mathcal{S}$, we have the following statements.

(1) $\mathrm{TC}_{n}$ holds if and only if $\mathrm{TC} 3_{n}$ holds.

(2) $\mathrm{TC}_{n}$ holds if and only if $\mathrm{TC}_{i}$ holds for all $i \leq n$.

(3) If $\mathrm{TC} 2_{n-1}$ and $\mathrm{TC} 1_{n}$ hold, then $\mathrm{TC} 2_{n}$ holds.

(4) GSC holds if and only if SDGSC holds, and this implies $\mathrm{TC}_{n}$ for all $n$.

Remark 3.3. Note that if we quantify over all $n$, the first three versions of the telescope conjecture are equivalent. That is,

$\mathrm{TC}_{n}$ for all $n \Longleftrightarrow \mathrm{TC} 2_{n}$ for all $n \Longleftrightarrow \mathrm{TC} 3_{n}$ for all $n$.

Remark 3.4. TC $2_{n}$ holds if and only if $L_{n}^{f} S^{0} \stackrel{\sim}{\rightarrow} L_{n} S^{0}$. Indeed, since both $L_{n}^{f}$ and $L_{n}$ are smashing, the subcategory of objects $W$ such that $L_{n}^{f} W \stackrel{\sim}{\rightarrow} L_{n} W$ is localizing. Thus if it contains $S^{0}$, it contains $\operatorname{loc}\left(S^{0}\right)=\mathcal{S}$.

Proof. First we show the equivalence of $\mathrm{TC}_{n}$ and $\mathrm{TC} 3_{n}$. This is also sketched in [Mahowald et al. 2001, 1.13]. For any type $n$ spectrum $Y, \operatorname{th}(Y)=\operatorname{th}(F(n))$ and so we have th $\left(L_{n} Y\right)=\operatorname{th}\left(L_{n} F(n)\right)$, and $\left\langle L_{n} Y\right\rangle=\left\langle L_{n} F(n)\right\rangle$. A construction in [Ravenel 1992, 8.3] gives a type $n$ spectrum $Y$ with $L_{n} Y \in \operatorname{th}(K(n))$. Thus $\left\langle L_{n} Y\right\rangle \leq\langle K(n)\rangle$, and $0 \neq\left\langle L_{n} F(n)\right\rangle=\langle K(n)\rangle$. Suppose $\mathrm{TC}_{n}$ holds. Then $\left\langle L_{n} Y\right\rangle=\left\langle f^{-1} Y\right\rangle=\langle T(n)\rangle$, and so $\langle T(n)\rangle=\langle K(n)\rangle$.

If $X$ is type $n$ and $f$ is a $v_{n}$ self-map, then [Mahowald and Sadofsky 1995, Proposition 3.2] implies that $L_{n}^{f} X \cong L_{T(n)} X \cong f^{-1} X$. Thus assuming TC $1_{n}$, we have $L_{K(n)} X \cong f^{-1} X$, and to prove $\mathrm{TC} 3_{n}$ it suffices to show that $L_{n} X \cong L_{K(n)} X$. This is known (see, e.g., [Hovey 1995a]), but we will give a proof that extends well to the localized setting. Since $\langle K(n)\rangle \leq\langle E(n)\rangle$, localization at $K(n)$ gives a map $L_{n} X \rightarrow L_{K(n)} X$. It suffices to show that this is an $L_{n}$-equivalence. The fiber is $K(n)$ acyclic, so $L_{n} X \wedge K(n) \rightarrow L_{K(n)} X \wedge K(n)$ is an isomorphism. Consider $i<n$. The triangle $C_{n} X \wedge K(i) \rightarrow X \wedge K(i) \rightarrow L_{n} X \wedge K(i)$ shows that $L_{n} X \wedge K(i)$ is zero, because $X$ is type $n$ and $C_{n} X$ is $K(i)$ acyclic. Lemma 3.3.1 in [Hovey et al. 1997] states that $L W=L S^{0} \wedge W$ for any localization $L$ and strongly dualizable $W$. Since every finite spectrum is strongly dualizable, $L_{K(n)} X \wedge K(i)=L_{K(n)} S^{0} \wedge X \wedge K(i)=0$. Thus $L_{n} X \wedge K(i) \rightarrow L_{K(n)} X \wedge K(i)$ is an isomorphism for all $i \leq n$, and hence $L_{n} X \rightarrow L_{K(n)} X$ is an $L_{n}$-equivalence.

For the second statement, we note that $\mathrm{TC} 2{ }_{n}$ is equivalent to the statement $\langle T(0) \vee \cdots \vee T(n)\rangle=\langle K(0) \vee \cdots \vee K(n)\rangle$. Smashing this with $\langle T(i)\rangle$, for $0 \leq i \leq n$, and using Lemma 2.10, yields $\mathrm{TC}_{i}$ for each $i$. The third statement is also clear from this observation.

Finally, GSC holds if and only if SDGSC holds because objects in $\mathcal{S}$ are compact if and only if they are strongly dualizable. Given GSC, consider $L_{n}$. The GSC 
would imply that the $L_{n}$-acyclics are $\operatorname{loc}(\langle E(n)\rangle \cap \mathcal{F})$. As observed earlier, this is the same as $\operatorname{loc}(F(n+1))$, the $L_{n}^{f}$-acyclics. Therefore we would have $L_{n}^{f} \cong L_{n}$.

The SDGSC is new, and we will discuss it first. As mentioned in the above proof, in $\mathcal{S}$ compactness is equivalent to strong dualizability. It is well known that localization away from a set of compact objects is smashing. The GSC is precisely the statement that the converse holds. However, as we will show next, one only needs strong dualizability to generate a smashing localization functor. We will state our result in slightly more general terms.

Theorem 3.5. Let $\mathrm{T}$ be a well generated tensor triangulated category such that $\operatorname{loc}(\mathbb{1})=\mathrm{T}$, as in Notation 2.1. Let $A=\left\{B_{\alpha}\right\}$ be a (possibly infinite) set of strongly dualizable objects. Then there exists a smashing localization functor $L: \mathrm{T} \rightarrow \mathrm{T}$ with $\operatorname{Ker} L=\operatorname{loc}(A)$.

Proof. Let $E=\vee_{\alpha} B_{\alpha}$ and note that $\operatorname{loc}(E)=\operatorname{loc}(A)$. The category $\mathrm{T}$ is well generated by hypothesis. The localizing subcategory $\mathrm{S}=\operatorname{loc}(E)$ is also well generated, by [Iyengar and Krause 2013, Remark 2.2], and is tensor-closed by Lemma 2.5.

By [Iyengar and Krause 2013, Proposition 2.1] there exists a localization functor $L: \mathrm{T} \rightarrow \mathrm{T}$ with $\operatorname{Ker} L=\mathrm{S}$. We will show that $L$ is a smashing localization.

First we claim that the $L$-locals are tensor-closed. For any $Y \in \mathrm{T}$, we have

$$
\begin{aligned}
Y \text { is } L \text {-local } & \Longleftrightarrow[W, Y]_{n}=0 \text { for all } W \in \mathrm{S} \text { and all } n \in \mathbb{Z} \\
& \Longleftrightarrow[E, Y]_{n}=\prod\left[B_{\alpha}, Y\right]_{n}=0 \text { for all } n \in \mathbb{Z} \\
& \Longleftrightarrow\left[B_{\alpha}, Y\right]_{n}=0 \text { for all } \alpha \text { and } n \in \mathbb{Z} \\
& \Longleftrightarrow D B_{\alpha} \wedge Y=0 \text { for all } \alpha .
\end{aligned}
$$

The second equivalence follows from the fact that $\left\{X \mid[X, Y]_{n}=0\right.$ for all $\left.n \in \mathbb{Z}\right\}$ is a localizing subcategory containing $E$, and hence all of $S$. The final equivalence uses the fact that the $B_{\alpha}$ are strongly dualizable.

Now suppose $Y$ is $L$-local and $X$ is arbitrary. Then $D B_{\alpha} \wedge Y=0$ for all $\alpha$, so $D B_{\alpha} \wedge Y \wedge X=0$ for all $\alpha$, and thus $Y \wedge X$ is $L$-local. This shows that the $L$-locals are tensor-closed.

Consider the localization triangle $C \mathbb{1} \rightarrow \mathbb{1} \rightarrow L \mathbb{1}$, where $L \mathbb{1}$ is $L$-local and $C \mathbb{1} \in \mathrm{S}$. For arbitrary $X \in \mathrm{T}$, tensoring gives an exact triangle,

$$
C \mathbb{1} \wedge X \rightarrow X \rightarrow L \mathbb{1} \wedge X
$$

The object $L \mathbb{1} \wedge X$ is $L$-local, since the locals are tensor-closed. Likewise, $C \mathbb{1} \wedge X \in \mathrm{S}$, since $S$ is tensor-closed and so $L(C \mathbb{1} \wedge X)=0$. Therefore $X \rightarrow L \mathbb{1} \wedge X$ is an $L$-equivalence from $X$ to an $L$-local object, and it follows that $L X \cong L \mathbb{1} \wedge X$. This shows that $L$ is a smashing localization. 
In the stable homotopy category, and more generally whenever $\mathbb{1} \in \mathrm{T}$ is compact, this gives nothing new; by [Hovey et al. 1997, Theorem 2.1.3(d)] compact and strongly dualizable are equivalent. Consider, however, the harmonic category, which has no nonzero compact objects. In Section 4 we classify all smashing localizations on the harmonic category; they are indexed by $\mathbb{N}$. Thus the GSC fails in the harmonic category but, as we show in Theorem 4.4, the SDGSC holds. In fact, in the following sections we will give several examples of categories where the GSC fails but the SDGSC holds.

On the other hand, we do not expect the SDGSC to hold in complete generality, since Keller's counterexample [1994] to the GSC is also a counterexample to the SDGSC; in the derived category of a ring $R$, the unit $R$ is compact and strongly dualizable, so the GSC and SDGSC are equivalent.

The GSC and SDGSC make sense in any localized category, but $\mathrm{TC} 1_{n}, \mathrm{TC} 2_{n}$, and $\mathrm{TC} 3_{n}$ may not, since $T(n)$ and $K(n)$ may not be objects in $\mathcal{L}$. Instead we present the following definitions.

Definition 3.6. Let $L: \mathcal{S} \rightarrow \mathcal{S}$ be a localization, and $\mathcal{L}$ the category of $L$-locals.

(1) Let $l_{n}^{f}: \mathcal{L} \rightarrow \mathcal{L}$ denote localization at $\langle L T(0) \vee L T(1) \vee \cdots \vee L T(n)\rangle$.

(2) Let $l_{n}: \mathcal{L} \rightarrow \mathcal{L}$ denote localization at $\langle L K(0) \vee L K(1) \vee \cdots \vee L K(n)\rangle$.

Before stating and proving a local version of Theorem 3.2, we establish some results about $l_{n}^{f}$ and $l_{n}$. First we make an observation about calculations in $\operatorname{BL}(\mathcal{L})$.

Lemma 3.7. All the calculations in Lemma 2.10 are valid in $\mathrm{BL}(\mathcal{L})$ if we replace $F(n), T(n)$, and $K(n)$ with $L F(n), L T(n)$, and $L K(n)$.

Proof. This follows from Theorem 2.3 and the statements in Lemma 2.10.

Proposition 3.8. The functor $l_{n}^{f}$ is localization away from $L F(n+1)$, and hence is smashing.

Proof. By Theorem 3.5 we know that there is some smashing localization functor $l: \mathcal{L} \rightarrow \mathcal{L}$ that is localization away from $L F(n+1)$; we wish to show $l=l_{n}^{f}$. Let $\mathbb{1}=L S^{0}$ for simplicity of notation, and let $c$ denote the colocalization corresponding to $l$. We claim that the $l$-acyclics are precisely $\operatorname{loc}(c \mathbb{1})$. Clearly $c \mathbb{1}$ is $l$-acyclic, and these are a localizing subcategory, so $\operatorname{loc}(c \mathbb{1}) \subseteq\{l$-acyclics $\}$. On the other hand, suppose $W$ is $l$-acyclic. Because $l$ is smashing, $L W=W \wedge l \mathbb{1}=0$, so $W=W \wedge c \mathbb{1}$. Then since $W \in \operatorname{loc}(\mathbb{1})=\mathcal{L}$, we have $W=W \wedge c \mathbb{1} \in \operatorname{loc}(\mathbb{1} \wedge c \mathbb{1})=\operatorname{loc}(c \mathbb{1})$, proving the claim.

By definition, the $l$-acyclics are also given by $\operatorname{loc}(L F(n+1))$. Therefore, we have $\langle L F(n+1)\rangle=\langle c \mathbb{1}\rangle$.

The class $\langle F(n+1)\rangle$ is complemented by $\langle T(0) \vee \cdots \vee T(n)\rangle$ in $\operatorname{BL}(\mathcal{S})$ [Hovey and Palmieri 1999, Section 5], and therefore $\langle L F(n+1)\rangle$ is complemented by 
$\langle L T(0) \vee \cdots \vee L T(n)\rangle$ in $\operatorname{BL}(\mathcal{L})$. At the same time, $\langle c \mathbb{1}\rangle$ is complemented by $\langle l \mathbb{1}\rangle$, and complements are unique. We conclude that $\{l$-acylics $\}=\langle l \mathbb{1}\rangle=$ $\langle L T(0) \vee \cdots \vee L T(n)\rangle$. Since $l$ and $l_{n}^{f}$ are two localizations on $\mathcal{L}$ with the same acyclics, they are equal.

Lemma 3.9. If $L$ is smashing, then $l_{n}^{f}=L L_{n}^{f}=L_{n}^{f} L$ and $l_{n}=L L_{n}=L_{n} L$, and both are smashing.

Proof. Smashing localization functors always commute, and they compose to give a smashing localization. The functor $L L_{n}: \mathcal{S} \rightarrow \mathcal{S}$, sending $X \mapsto L\left(L_{n} S^{0} \wedge X\right)=$ $L S^{0} \wedge L_{n} S^{0} \wedge X$ is a smashing localization. Since $L L_{n}$-locals are $L$-local, it also gives a smashing localization on $\mathcal{L}$. The acyclics of this functor are $\left\langle L L_{n} S^{0}\right\rangle$ in $\operatorname{BL}(\mathcal{L})$, which is $\langle L K(0) \vee \cdots \vee L K(n)\rangle$. Thus $L L_{n}$ and $l_{n}$ are localizations on $\mathcal{L}$ with the same acyclics, and hence isomorphic. The same proof works for $l_{n}^{f}=L L_{n}^{f}$.

In the category $\mathcal{S}$, for a type $n$ finite spectrum $X$ with a $v_{n}$ map $f: \Sigma^{d} X \rightarrow X$ and telescope $f^{-1} X$, it is known [Mahowald and Sadofsky 1995, Proposition 3.2] that $L_{n}^{f} X \cong L_{T(n)} X \cong f^{-1} X$. The following proposition shows that the local version of this result holds as well.

Lemma 3.10. Let $L: \mathcal{S} \rightarrow \mathcal{S}$ be a localization, and $l_{n}^{f}, X$ and $f^{-1} X$ as above. Then

$$
l_{n}^{f}(L X) \cong L_{L T(n)}(L X) \cong L\left(f^{-1} X\right) .
$$

Proof. The proof parallels the [Mahowald and Sadofsky 1995] result; one must only check that everything works when localized. If $L_{L T(n)}(L X) \cong L\left(f^{-1} X\right)$ holds for a single type $n$ spectrum, then it holds for all type $n$ spectra. So without loss of generality, we can choose $X$ to be a type $n$ spectrum that is a ring object in $\mathcal{S}$. Then for any $v_{n}$ self-map $f$, the telescope $f^{-1} X$ is also a ring object [Mahowald and Sadofsky 1995, Lemma 2.2]. By Lemma 2.5, $L\left(f^{-1} X\right)$ is a ring object in $\mathcal{L}$, and hence is local with respect to itself.

Lemma 2.2 in [Mahowald and Sadofsky 1995] shows that we have $X \wedge f^{-1} X \cong$ $f^{-1} X \wedge f^{-1} X$ in $\mathcal{S}$, so $L X \wedge_{\mathcal{L}} L\left(f^{-1} X\right) \cong L\left(f^{-1} X\right) \wedge_{\mathcal{L}} L\left(f^{-1} X\right)$ in $\mathcal{L}$ and the canonical map $L X \rightarrow L\left(f^{-1} X\right)$ is an $L\left(f^{-1} X\right)$-equivalence. It follows that $L_{L T(n)}(L X) \cong L\left(f^{-1} X\right)$.

Since $\langle L T(n)\rangle \leq\langle L T(0) \vee \cdots \vee L T(n)\rangle$, we have that $L\left(f^{-1} X\right)$ is $l_{n}^{f}$-local. One then uses Lemma 3.7 to see that $L X \rightarrow L\left(f^{-1} X\right)$ is a $l_{n}^{f}$-equivalence, and so $l_{n}^{f}(L X)=l_{n}^{f}\left(L\left(f^{-1} X\right)\right)=L\left(f^{-1} X\right)$.

Definition 3.11. Let $L: \mathcal{S} \rightarrow \mathcal{S}$ be a localization, and consider the category $\mathcal{L}$ of locals. Fix an $n \geq 0$. We have the following versions of the telescope conjecture on $\mathcal{L}$. 
$\mathrm{LTC}_{n} \quad\langle L T(n)\rangle=\langle L K(n)\rangle$.

$\mathrm{LTC}_{n} \quad l_{n}^{f} X \stackrel{\sim}{\rightarrow} l_{n} X$ for all $X$.

LTC $3_{n} \quad$ If $X \in \mathcal{S}$ is type $n$ and $f$ is a $v_{n}$ self-map, then $l_{n}(L X) \cong L\left(f^{-1} X\right)$.

GSC Every smashing localization is generated by a set of compact objects.

SDGSC Every smashing localization is generated by a set of strongly dualizable objects.

Theorem 3.12. On the category $\mathcal{L}$, we have the following statements.

(1) $\mathrm{LTC}_{n}$ implies $\mathrm{LTC} 3_{n}$.

(2) $\mathrm{LTC}_{n}$ holds if and only if $\mathrm{LTC}_{i}$ holds for all $i \leq n$.

(3) If $\mathrm{LTC}_{2-1}$ and $\mathrm{LTC} 1_{n}$ hold, then $\mathrm{LTC} 2_{n}$ holds.

Proof. Note that $\mathrm{LTC}_{n}$ is equivalent to the statement $\langle L T(0) \vee \cdots \vee L T(n)\rangle=$ $\langle L K(0) \vee \cdots \vee L K(n)\rangle$, so the last two statements are clear. We will show that $\mathrm{LTC}_{n}$ implies $\mathrm{LTC}_{n}$ by mimicking the proof in Theorem 3.2.

If $X$ is type $n$ and $f$ is a $v_{n}$ self-map, Lemma 3.10 shows that $l_{n}^{f}(L X) \cong$ $L_{L T(n)}(L X) \cong L\left(f^{-1} X\right)$. Then $\mathrm{LTC1}_{n}$ implies $L_{L K(n)} L X \cong L\left(f^{-1} X\right)$. So it suffices to show that $l_{n}(L X)=L_{L K(n)} L X$. Now we must show that the map $L_{L K(n)}: l_{n}(L X) \rightarrow L_{L K(n)} L X$ is an $l_{n}$-equivalence. The same reasoning as in Theorem 3.2, along with the computations of Lemma 3.7 and some definition unwinding, gives us that $l_{n}(L X) \wedge L K(i) \rightarrow L_{L K(n)} L X \wedge L K(i)$ is an equivalence for all $i \leq n$; we only need to notice that Lemma 3.3.1 in [Hovey et al. 1997] applies to strongly dualizable objects, and that $L X$ is strongly dualizable.

Theorem 3.13. If, furthermore, $L: \mathcal{S} \rightarrow \mathcal{S}$ is a smashing localization, then on the category $\mathcal{L}$ of locals we have that $\mathrm{LTC}_{n}$ implies $\mathrm{LTC} 1_{n}$, and

$$
\mathrm{GSC} \Longleftrightarrow \mathrm{SDGSC} \Rightarrow \mathrm{LTC}_{n} \text { for all } n \text {. }
$$

Remark 3.14. In this case, $\mathrm{LTC} 2_{n}$ is equivalent to $l_{n}^{f}\left(L S^{0}\right) \stackrel{\sim}{\rightarrow} l_{n}\left(L S^{0}\right)$, since by Lemma 3.9 both $l_{n}^{f}$ and $l_{n}$ are smashing, so the argument in Remark 3.4 applies.

Proof. By [Hovey et al. 1997, Theorem 2.1.3(d)], the compact objects and strongly dualizable objects in $\mathcal{L}$ coincide. Thus GSC holds if and only if SDGSC holds, and this implies $\mathrm{LTC}_{n}$ just as in Theorem 3.2.

Suppose $X$ has type $n$ and $\mathrm{LTC}_{n}$ holds. As in the proof of Theorem 3.2, $\left\langle L_{n} F(n)\right\rangle=\langle K(n)\rangle$ in $\operatorname{BL}(\mathcal{S})$, and so $\left\langle L L_{n} L F(n)\right\rangle=\langle L K(n)\rangle$ in $\operatorname{BL}(\mathcal{L})$. By Lemma 3.9, we have $\left\langle l_{n} L F(n)\right\rangle=\langle L K(n)\rangle$. Then $\mathrm{LTC}_{n}$ implies that $\langle L T(n)\rangle=$ $\left\langle L\left(f^{-1} X\right\rangle=\left\langle l_{n}(L X)\right\rangle=\left\langle l_{n} L F(n)\right\rangle\right.$, so $\mathrm{LTC}_{n}$ holds.

Question 3.15. Is $l_{n}: \mathcal{L} \rightarrow \mathcal{L}$ always a smashing localization?

This is the case in all the local categories investigated in this paper, whether or not $L: \mathcal{S} \rightarrow \mathcal{S}$ is a smashing localization. If one could show $l_{n}$ is always smashing, then most likely on $\mathcal{L}$ one would have that SDGSC implies $\mathrm{LTC} 2_{n}$ for all $n$. 
We would of course like to know if and when information on localized telescope conjectures can help with those in the original category $\mathcal{S}$, where all versions remain open.

Proposition 3.16. Let $L: \mathcal{S} \rightarrow \mathcal{S}$ be a localization, with localized category $\mathcal{L}$.

(1) If $\mathrm{TC} 1_{n}$ holds on $\mathcal{S}$, then $\mathrm{LTC}_{n}$ holds on $\mathcal{L}$.

(2) If $\mathrm{TC} 2_{n}$ holds on $\mathcal{S}$, then $\mathrm{LTC} 2_{n}$ holds on $\mathcal{L}$.

(3) If $\mathrm{TC} 3_{n}$ holds on $\mathcal{S}$, then $\mathrm{LTC} 3_{n}$ holds on $\mathcal{L}$.

Furthermore, if $L$ is a smashing localization, then we have the following.

(4) If GSC holds on $\mathcal{S}$, then GSC holds on $\mathcal{L}$.

(5) If SDGSC holds on $\mathcal{S}$, then SDGSC holds on $\mathcal{L}$.

Proof. Part (1) follows immediately from Proposition 2.6. So does Part (2), since $\mathrm{TC} 2{ }_{n}$ is equivalent to the statement $\langle T(0) \vee \cdots \vee T(n)\rangle=\langle K(0) \vee \cdots \vee K(n)\rangle$, and similarly for $\mathrm{LTC}_{n}$. From this and Theorems 3.2 and 3.12 we have

$$
\mathrm{TC}_{n} \Longleftrightarrow \mathrm{TC}_{n} \Rightarrow \mathrm{LTC}_{n} \Rightarrow \mathrm{LTC}_{n} .
$$

Now suppose $L$ is smashing and the GSC holds on $\mathcal{S}$. Let $l: \mathcal{L} \rightarrow \mathcal{L}$ be a smashing localization. Thus $l$ is defined by $l(L Y)=l\left(L S^{0}\right) \wedge_{\mathcal{L}} L Y=l S^{0} \wedge L S^{0} \wedge Y$. We can therefore extend $l$ to be a smashing localization on all of $\mathcal{S}$, with the map $X \mapsto l S^{0} \wedge L S^{0} \wedge X=l L S^{0} \wedge X$. Since the GSC holds on $\mathcal{S}$ by assumption, the acyclics of this functor are $\left\langle l L S^{0}\right\rangle=\operatorname{loc}(A)$, for some set of compact objects $A$ in $\mathcal{S}$. Here $\left\langle l L S^{0}\right\rangle$ refers to the Bousfield class in $\operatorname{BL}(\mathcal{S})$.

We must show that $\left\langle l L S^{0}\right\rangle$ in $\operatorname{BL}(\mathcal{L})$ is generated by a set of objects that are compact in $\mathcal{L}$. Note that $\left\langle l L S^{0}\right\rangle$ in $\operatorname{BL}(\mathcal{L})$ is $\left\{L W \mid L W \wedge_{\mathcal{L}} l L S^{0}=0\right\}=$ $\left\{L W \mid L W \wedge_{\mathcal{S}} l L S^{0}=0\right\}=\left\langle l L S^{0}\right\rangle \cap \mathcal{L}$, where the latter $\left\langle l L S^{0}\right\rangle$ is in $\operatorname{BL}(\mathcal{S})$. Therefore $\left\langle l L S^{0}\right\rangle$ in $\operatorname{BL}(\mathcal{L})$ is $\operatorname{loc}(A) \cap \mathcal{L}$. We claim that this is $\operatorname{loc}(L(A))$. Since $L$ sends compacts to compacts, this will show that $l$ is generated by a set of compacts.

If $X \in \operatorname{loc}(A)$, then $L X \in \operatorname{loc}(L(A))$. If $X \in \mathcal{L}$ in addition, then we have $X \cong L X \in \operatorname{loc}(L(A))$. For the other inclusion, note that the intersection of two localizing subcategories is a localizing subcategory, and $\mathcal{L}$ is a localizing subcategory of $\mathcal{S}$ because $L$ is smashing. For $Y \in A, L Y$ is in $\mathcal{L}$, and $L Y=L S^{0} \wedge Y \in \operatorname{loc}(Y) \subseteq$ $\operatorname{loc}(A)$. Therefore $L(A) \subseteq \operatorname{loc}(A) \cap \mathcal{L}$, and $\operatorname{loc}(L(A))=\operatorname{loc}(A) \cap \mathcal{L}$.

Part (5) follows immediately, since if $L$ is smashing then GSC holds if and only if SDGSC in both $\mathcal{S}$ and $\mathcal{L}$.

Balmer and Favi [2011, Proposition 4.4] have also recently proved Part (4) in the slightly more general setting of a smashing localization on a unital algebraic stable homotopy category; the above proof would apply there as well. One would 
like to prove Part (5) without the assumption that $L$ is smashing, but it's not clear if this is possible.

Letting $L=L_{Z}$, for $Z=\bigvee_{i \geq 0} K(i), E(n), K(n), B P, H \mathbb{F}_{p}$, or $I$ provides interesting examples of categories $\mathcal{L}$ on which to investigate these telescope conjectures. Furthermore, $\mathrm{LTC1}_{n}$ suggests the relevance of Bousfield lattices to understanding these questions. In the remaining sections, we focus on specific localized categories.

\section{The Harmonic category}

Let $Q=\bigvee_{i \geq 0} K(i)$ and $L=L_{Q}: \mathcal{S} \rightarrow \mathcal{S}$, and consider the harmonic category $\mathcal{H}$ of $L$-locals. Harmonic localization is not smashing. An object is called harmonic if it is $L$-local and dissonant if it is $L$-acyclic. For example, finite spectra, suspension spectra, finite torsion spectra, and BP are known to be harmonic [Hovey 1995a; Ravenel 1984]. On the other hand, $I$ and $H \mathbb{F}_{p}$ are dissonant.

In order to answer the telescope conjectures in $\mathcal{H}$, we will first calculate the Bousfield lattice of $\mathcal{H}$. In this section all smash products are in $\mathcal{H}$ unless otherwise noted. Given any set $P$, let $2^{P}$ denote the power set of $P$.

Definition 4.1. Given $X \in \mathcal{H}$, define the support of $X$ to be

$$
\operatorname{supp}(X)=\{i \mid X \wedge K(i) \neq 0\} \subseteq \mathbb{N} .
$$

The following result and proof was pointed out to us by Jon Beardsley.

Proposition 4.2. The Bousfield lattice of $\mathcal{H}$ is $2^{\mathbb{N}}$.

Proof. Each $K(n)$ is a ring object, and hence $K(n)$-local by Lemma 2.5. Because $\langle K(n)\rangle \leq\langle Q\rangle, K(n)$-locals are harmonic, thus each $K(n)$ is harmonic. The argument hinges on the fact that $K(n)$ is a skew field object in $\mathcal{H}$ : for $X=L X$ in $\mathcal{H}$, if $X \wedge K(n) \neq 0$ then $X \wedge K(n)=L\left(X \wedge_{\mathcal{S}} K(n)\right)$ so $X \wedge_{\mathcal{S}} K(n) \neq 0$, and $X \wedge_{\mathcal{S}} K(n)$ must be a nonempty wedge of suspensions of $K(n)$ s. Thus

$$
\begin{aligned}
X \wedge K(n) & =L(X \wedge \mathcal{S} K(n))=L\left(\vee \Sigma^{i} K(n)\right)=L\left(\vee \Sigma^{i} L K(n)\right) \\
& =\coprod_{\mathcal{L}} \Sigma^{i} L K(n)=\coprod_{\mathcal{L}} \Sigma^{i} K(n) .
\end{aligned}
$$

It follows that $L X \wedge K(n)=0$ if and only if $L X \wedge_{\mathcal{S}} K(n)=0$. Furthermore, if $L X \wedge K(n) \neq 0$, then $\langle L X \wedge K(n)\rangle=\langle K(n)\rangle$, where these are Bousfield classes in $\operatorname{BL}(\mathcal{H})$.

By the definition of $L$, for any $W \in \mathcal{S}$, if $W \wedge_{\mathcal{S}} K(n)=0$ for all $n$, then $L W=0$. Combining this with the above observation, we get that a local object $W=L W$ has $W \wedge K(n)=0$ in $\mathcal{H}$ for all $n$ if and only if $W=0$.

Therefore, for any $X, Y \in \mathcal{H}$, we have

$Y \wedge X=0 \Longleftrightarrow Y \wedge X \wedge K(n)=0$ for all $n \Longleftrightarrow Y \wedge K(n)=0$ for all $n \in \operatorname{supp}(X)$. 
We conclude that there is a lattice isomorphism $F: \operatorname{BL}(\mathcal{H}) \rightarrow 2^{\mathbb{N}}$, given by

$$
\langle X\rangle=\bigvee_{\operatorname{supp}(X)}\langle K(i)\rangle \mapsto \operatorname{supp}(X), \quad N \subseteq \mathbb{N} \mapsto \bigvee_{i \in N}\langle K(i)\rangle
$$

Theorem 4.3. On $\mathcal{H}$, for all $n \geq 0$, we have that $\mathrm{LTC}_{n}, \mathrm{LTC}_{n}$, and $\mathrm{LTC} 3_{n}$ hold.

Proof. By Lemmas 2.10 and 3.7, $L T(n)$ and $L K(n)=K(n)$ have the same support. The above theorem then implies that $\langle L T(n)\rangle=\langle L K(n)\rangle$. Thus $\mathrm{LTC}_{n}$ holds for all $n$, and the claim follows from Theorem 3.12.

Next, we classify all smashing localizations on $\mathcal{H}$, and show that the GSC fails but the SDGSC holds. The proof is based on that of [Hovey and Strickland 1999, Theorem 6.14], which classifies smashing localizations in the $E(n)$-local category.

Theorem 4.4. If $L^{\prime}: \mathcal{H} \rightarrow \mathcal{H}$ is a smashing localization functor, then $L^{\prime}=l_{n}^{f}$ for some $n \geq 0$, or $L^{\prime}=0$ or $L^{\prime}=\mathrm{id}$. Therefore the GSC fails but the SDGSC holds on $\mathcal{H}$.

Proof. Let $L^{\prime}: \mathcal{H} \rightarrow \mathcal{H}$ be a smashing localization functor, and let $\mathbb{1}=L S^{0}$ be the unit in $\mathcal{H}$. The acyclics of $L^{\prime}$ are given by $\left\langle L^{\prime} \mathbb{1}\right\rangle$. From Proposition $4.2,\left\langle L^{\prime} \mathbb{1}\right\rangle$ is equal to the wedge of $\langle K(i)\rangle$ for all $i \in \operatorname{supp}\left(L^{\prime} \mathbb{1}\right)$. If $\operatorname{supp}\left(L^{\prime} \mathbb{1}\right)=\varnothing$ then $\left\langle L^{\prime} \mathbb{1}\right\rangle=\langle 0\rangle$ and $L^{\prime}=0$.

Assume now that $\operatorname{supp}\left(L^{\prime} \mathbb{1}\right)$ is not empty, and take $j \in \operatorname{supp}\left(L^{\prime} \mathbb{1}\right)$. We will show that $\left\langle L^{\prime} \mathbb{1}\right\rangle \geq\langle K(0) \vee \cdots \vee K(j)\rangle$. It follows that either $\left\langle L^{\prime} \mathbb{1}\right\rangle=\bigvee_{i \geq 0}\langle K(i)\rangle=\langle\mathbb{1}\rangle$ and $L^{\prime}=\mathrm{id}$, or $L^{\prime}=l_{n}=l_{n}^{f}$ for $n=\max \left(\operatorname{supp}\left(L^{\prime} \mathbb{1}\right)\right)$.

Since $\langle K(j)\rangle \leq\left\langle L^{\prime} \mathbb{1}\right\rangle$, from Lemma 2.8 we have $L_{K(j)} L^{\prime}=L^{\prime} L_{K(j)}=L_{K(j)}$. Therefore $\left\langle L_{K(j)} \mathbb{1}\right\rangle=\left\langle L^{\prime} \mathbb{1} \wedge L_{K(j)} \mathbb{1}\right\rangle \leq\left\langle L^{\prime} \mathbb{1}\right\rangle$. Proposition 5.3 of [Hovey and Strickland 1999] shows that, in $\mathcal{S}, L_{K(j)} S^{0} \wedge_{\mathcal{S}} K(i)$ is nonzero for $0 \leq i \leq j$ and zero for $i>j$. Note that $L_{K(j)} S^{0}=L_{K(j)} L S^{0}=L_{K(j)} \mathbb{1}$ and, as remarked in the proof of Proposition 4.2, $L X \wedge K(i)=0$ if and only if $L X \wedge_{\mathcal{S}} K(i)=0$. Therefore, in BL(H) we have $\left\langle L_{K(j)} \mathbb{1}\right\rangle=\langle K(0) \vee \cdots \vee K(j)\rangle$, and so $\left\langle L^{\prime} \mathbb{1}\right\rangle \geq\langle K(0) \vee \cdots \vee K(j)\rangle$ as desired.

Each $l_{n}^{f}$ is localization away from $L F(n+1)$ by Proposition 3.8, which is strongly dualizable by Theorem 2.3. The identity is localization away from zero, and the zero functor is localization away from $L S^{0}$; these are both strongly dualizable. Therefore the SDGSC holds. On the other hand, Corollary B.13 in [Hovey and Strickland 1999] shows that there are no nonzero compact objects in $\mathcal{H}$, so the GSC fails.

Question 4.5. Classify localizing subcategories of $\mathcal{H}$.

It seems likely that every localizing subcategory of $\mathcal{H}$ is a Bousfield class, and so these are in bijection with $2^{\mathbb{N}}$, but we have been unable to prove this. 


\section{The $E(n)$ - and $K(n)$ - local categories}

5A. The E(n)-local category. Recall that $\langle E(n)\rangle=\langle K(0) \vee K(1) \vee \cdots \vee K(n)\rangle$. In this section, fix $L=L_{n}=L_{E(n)}: \mathcal{S} \rightarrow \mathcal{S}$ and let $\mathcal{L}_{n}$ denote the local category. The functor $L_{n}$ is smashing, and so by Notation 2.1 each $L F(i)$ is compact in $\mathcal{L}_{n}$. Hovey and Strickland [1999] have studied $\mathcal{L}_{n}$ in detail and determined the localizing subcategories, smashing localizations, and Bousfield lattice of $\mathcal{L}_{n}$. We begin by recalling these results.

Lemma 5.1. For $0 \leq i \leq n$ we have $L K(i)=K(i)$, and for $i>n$ we have $L K(i)=0$. Proof. This follows from $\langle E(n)\rangle=\langle K(0) \vee K(1) \vee \cdots \vee K(n)\rangle$.

Theorem 5.2 [Hovey and Strickland 1999, Theorem 6.14]. The lattice of localizing subcategories of $\mathcal{L}_{n}$, ordered by inclusion, is in bijection with the lattice of subsets of the set $\{0,1, \ldots, n\}$, where a localizing subcategory $\mathrm{S}$ corresponds to

$$
\{i \mid K(i) \in \mathrm{S}\} .
$$

Corollary 5.3. Every localizing subcategory of $\mathcal{L}_{n}$ is a Bousfield class, in particular a localizing subcategory $\mathrm{S}$ is the Bousfield class

$$
\bigvee\langle K(j) \mid K(j) \notin \mathrm{S}, 0 \leq j \leq n\rangle .
$$

Corollary 5.4. For every $n \geq 0$, there is a lattice isomorphism

$$
f_{n}: \operatorname{BL}\left(\mathcal{L}_{n}\right) \stackrel{\sim}{\longrightarrow} 2^{\{0,1, \ldots, n\}} .
$$

Proof. The isomorphism is given by

$$
\begin{gathered}
\langle X\rangle=\bigvee_{X \wedge K(i) \neq 0}\langle K(i)\rangle \mapsto\{i \mid X \wedge K(i) \neq 0\}, \\
N \subseteq\{0,1, \ldots, n\} \mapsto \bigvee_{i \in N}\langle K(i)\rangle .
\end{gathered}
$$

Theorem 5.5 [Hovey and Strickland 1999, Corollary 6.10]. If $L^{\prime}: \mathcal{L}_{n} \rightarrow \mathcal{L}_{n}$ is a smashing localization, then $L^{\prime}=L_{i}=L_{i}^{f}$ for some $0 \leq i \leq n$ or $L^{\prime}=0$. Thus the GSC holds on $\mathcal{L}_{n}$.

Corollary 5.6. On $\mathcal{L}_{n}$, all of $\mathrm{LTC} 1_{i}, \mathrm{LTC}_{i}, \mathrm{LTC} 3_{i}$ hold for all $i$, and $\mathrm{GSC}$ and SDGSC also hold.

Proof. This follows from Theorems 5.5 and 3.13. Note that for $i>n$, we have $L T(i)=0=L K(i)$ by Lemma 2.10 , and so $l_{i}=l_{n}=l_{n}^{f}=l_{i}^{f}$.

Recall that there is a natural map $L_{n} X \rightarrow L_{n-1} X$ for all $X$ in $\mathcal{S}$ and $n$, and by Proposition 2.6 this induces a surjective lattice map $\operatorname{BL}\left(\mathcal{L}_{n}\right) \rightarrow \operatorname{BL}\left(\mathcal{L}_{n-1}\right)$ and an inverse system of lattice maps.

$$
\cdots \rightarrow \mathrm{BL}\left(\mathcal{L}_{n}\right) \rightarrow \mathrm{BL}\left(\mathcal{L}_{n-1}\right) \rightarrow \cdots \rightarrow \operatorname{BL}\left(\mathcal{L}_{1}\right) \rightarrow \operatorname{BL}\left(\mathcal{L}_{0}\right)
$$


Proposition 5.7. The lattice isomorphisms $F$ and $f_{n}$ from Proposition 4.2 and Corollary 5.4 realize $\mathrm{BL}(\mathcal{H})$ as the inverse limit of the maps $\mathrm{BL}\left(\mathcal{L}_{n}\right) \rightarrow \operatorname{BL}\left(\mathcal{L}_{n-1}\right)$.

Proof. By Lemma 2.8 and the facts that $L_{Q} K(i)=K(i)$ for all $i$, and $L_{n} K(i)=K(i)$ for $i \leq n$ and $L_{n} K(i)=0$ for $i>n$, we get the following diagram for all $n$. The map $2^{\{0,1, \ldots, n\}} \rightarrow 2^{\{0,1, \ldots, n-1\}}$ is induced by sending $m \mapsto m$ for $m<n$ but $n \mapsto 0$, and the maps $2^{\mathbb{N}} \rightarrow 2^{\{0,1, \ldots, i\}}$ are defined similarly.

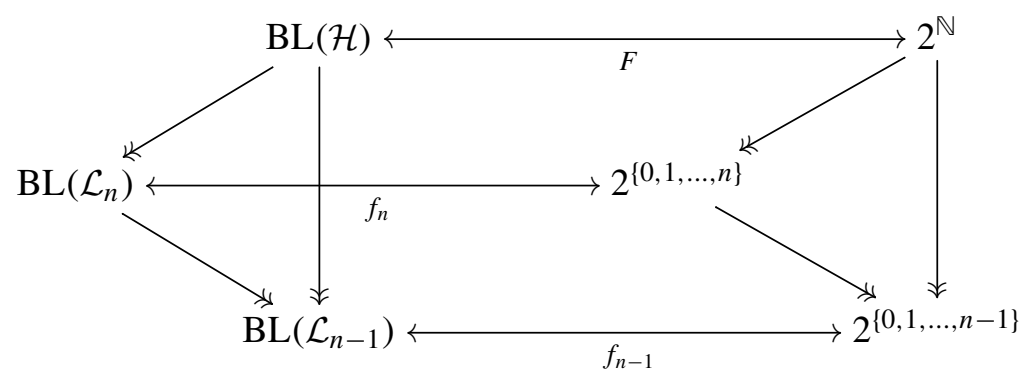

5B. The K(n)-local category. Although an incredibly complicated category in its own right, the $K(n)$-local category is quite basic from the perspective of localizing subcategories, Bousfield lattices, and telescope conjectures. In this subsection, let $L=L_{K(n)}: \mathcal{S} \rightarrow \mathcal{S}$ be localization at $K(n)$, and let $\mathcal{K}_{n}$ denote the category of locals. Hovey and Strickland classify the localizing subcategories of $\mathcal{K}_{n}$, and there are not many of them.

Proposition 5.8 [Hovey and Strickland 1999, Theorem 7.5]. There are no nonzero proper localizing subcategories of $\mathcal{K}_{n}$.

This Proposition implies that the Bousfield lattice of $\mathcal{K}_{n}$ is the two-element lattice $\{\langle 0\rangle,\langle K(n)\rangle\}$. We will prove a slightly more general result that will be used again in Section 6A.

Proposition 5.9. Consider a category $\mathrm{T}$ as in Notation 2.1, an object $Z$ in $\mathrm{T}$, and localization $L_{Z}: \mathrm{T} \rightarrow \mathrm{T}$ with localized category $\mathcal{L}_{Z}$.

(1) If $Z$ is a ring object, then $\left\langle L_{Z} Z\right\rangle=\langle Z\rangle$ is the maximum class in $\operatorname{BL}\left(\mathcal{L}_{Z}\right)$.

(2) If $Z$ is a skew field object, then $\operatorname{BL}\left(\mathcal{L}_{Z}\right)$ is the two-element lattice $\{\langle 0\rangle,\langle Z\rangle\}$.

Proof. For (1), note that Lemma 2.5 implies $L_{Z} Z=Z$. Consider $\langle Z\rangle$ in $\operatorname{BL}\left(\mathcal{L}_{Z}\right)$. By definition, this is the collection of all $W \in \mathcal{L}_{Z}$ with $L\left(Z \wedge_{\mathrm{T}} W\right)=0$. But $Z \wedge_{\mathrm{T}} W$ is a $Z$-module object in $T$, and hence $L_{Z}$-local. The only object that is both local and acyclic with respect to any localization is zero, so $Z \wedge_{\mathrm{T}} W=0$. But this says that $W$ is $L_{Z}$-acyclic, and hence zero in $\mathcal{L}_{Z}$. Therefore, in $\operatorname{BL}\left(\mathcal{L}_{Z}\right)$ we have $\langle Z\rangle=\{0\}$.

Now suppose $Z$ is a skew field object in $T$. In particular, it is a ring object, so $\langle Z\rangle$ is the maximum class in $\operatorname{BL}\left(\mathcal{L}_{Z}\right)$. Consider $\langle L X\rangle$ in $\operatorname{BL}\left(\mathcal{L}_{Z}\right)$, for arbitrary $X \in \mathrm{T}$. If $X \wedge_{\mathrm{T}} Z=0$, then $L X=0$. Otherwise, $X \wedge_{\mathrm{T}} Z$ is a wedge of suspensions 
of $Z$, so $\langle Z\rangle=\left\langle X \wedge_{\mathrm{T}} Z\right\rangle \leq\langle X\rangle$ in $\operatorname{BL}(\mathrm{T})$. Then $\langle Z\rangle=\left\langle L_{Z} Z\right\rangle \leq\left\langle L_{Z} X\right\rangle$ in $\operatorname{BL}\left(\mathcal{L}_{Z}\right)$, so $\left\langle L_{Z} X\right\rangle=\langle Z\rangle$.

Corollary 5.10. The Bousfield lattice of $\mathcal{K}_{n}$ is $\{\langle 0\rangle,\langle K(n)\rangle\}$.

Theorem 5.11. In $\mathcal{K}_{n}$, all of $\mathrm{LTC}_{i}$, $\mathrm{LTC} 2_{i}$, $\mathrm{LTC} 3_{i}$ hold for all $i$, and $\mathrm{GSC}$ and SDGSC also hold.

Proof. In light of Theorem 3.12, we will show that $\mathrm{LTC}_{i}$ holds for all $i$. This follows from Lemma 2.10: for $i \neq n$ we have $L T(i)=0=L K(i)$, but $L T(n) \neq 0$ so by the last corollary $\langle L T(n)\rangle=\langle K(n)\rangle=\langle L K(n)\rangle$.

There are exactly two smashing localizations on $\mathcal{K}_{n}$. The identity functor is smashing and is localization away from 0 , which is compact and strongly dualizable. The zero functor is smashing and is localization away from $L S^{0}$, which is strongly dualizable. It is not compact, but by Theorem 7.3 in [Hovey and Strickland 1999] $L F(n)$ is compact in $\mathcal{K}_{n}$ and $\operatorname{loc}(L F(n))=\operatorname{loc}\left(L S^{0}\right)=\mathcal{K}_{n}$. Therefore the zero functor is also generated by a compact object. This shows that both the GSC and SDGSC hold.

\section{Other localized categories}

In this section we will consider several other localized categories. In each case, let $L_{Z}: \mathcal{S} \rightarrow \mathcal{S}$ denote the localization functor that annihilates $\langle Z\rangle$, and let $\mathcal{L}_{Z}$ denote the category of $L_{Z}$-locals.

6A. The $\boldsymbol{H} \mathbb{F}_{\boldsymbol{p}}$-local category. The Eilenberg-MacLane spectrum $H \mathbb{F}_{p}$ is a skew field object in $\mathcal{S}$; in fact, every skew field object in $\mathcal{S}$ is isomorphic to either $H \mathbb{F}_{p}$ or a $K(n)$. Unlike the $\langle K(n)\rangle$, it is not complemented; for example, $\langle I\rangle \leq\left\langle H \mathbb{F}_{p}\right\rangle$ but $I \wedge H \mathbb{F}_{p}=0$. So $\left\langle H \mathbb{F}_{p}\right\rangle \in \mathrm{DL} \backslash \mathrm{BA}$. Hovey and Palmieri [1999] have conjectured several results about the collection of classes less than $\left\langle H \mathbb{F}_{p}\right\rangle$ in $\operatorname{BL}(\mathcal{S})$. The telescope conjectures and Bousfield lattice of $\mathcal{L}_{H \mathbb{F}_{p}}$ are quite simple.

Theorem 6.1. In $\mathcal{L}_{H \mathbb{F}_{p}}$, all of $\mathrm{LTC}_{n}, \mathrm{LTC}_{n}, \mathrm{LTC}_{n}$ hold for all $n$.

Proof. For all $n, K(n) \wedge H \mathbb{F}_{p}=0$ and $T(n) \wedge H \mathbb{F}_{p}=0$, by [Hovey and Palmieri 1999, p. 16]. Therefore $L K(n)=0=L T(n)$ and $\mathrm{LTC}_{n}$ holds for all $n$. Note that $l_{n}=l_{n}^{f}$ is the zero functor for all $n$.

In order to discuss the GSC and SDGSC in this category, we must classify the smashing localizations. We will do this by using what we know about the Bousfield lattice.

Proposition 6.2. The Bousfield lattice of $\mathcal{L}_{H \mathbb{F}_{p}}$ is the two-element lattice $\left\{\langle 0\rangle,\left\langle H \mathbb{F}_{p}\right\rangle\right\}$. Proof. This follows immediately from Proposition 5.9 because $H \mathbb{F}_{p}$ is a skew field object in $\mathcal{S}$. 
Recall that every smashing localization gives a pair of complemented classes in $\mathrm{BA} \subseteq \mathrm{BL}$. Thus in $\mathcal{L}_{H \mathbb{F}_{p}}$ there are exactly two smashing localizations, the trivial ones given by smashing with zero and with the unit.

Proposition 6.3. In $\mathcal{L}_{H \mathbb{F}_{p}}$, the GSC fails but the SDGSC holds.

Proof. The identity functor is smashing and is localization away from 0 , which is compact and strongly dualizable. By [Hovey and Strickland 1999, Corollary B. 13], there are no nonzero compact objects in $\mathcal{L}_{H \mathbb{F}_{p}}$. So the zero functor, which is localization away from $L S^{0}$, is generated by a strongly dualizable object but not a compact one.

One application of this Bousfield lattice calculation is to the question of classifying localizing subcategories. Every Bousfield class is a localizing subcategory. Hovey and Palmieri [1999, Conjecture 9.1] suggest that the converse holds in the $p$-local stable homotopy category. The original conjecture is still open, but the question can be asked in any well-generated tensor triangulated category. For example, in a stratified category every localizing subcategory is a Bousfield class. The question is interesting, since in general localizing subcategories are hard to classify. In many cases, including $\mathcal{S}$, it is not even known if there is a set of localizing subcategories. Recently Stevenson [2014] found the first counterexample, in an algebraic setting: in the derived category of an absolutely flat ring that is not semiartinian, there are localizing subcategories that are not Bousfield classes. Now we show that $\mathcal{L}_{H \mathbb{F}_{p}}$ provides another counterexample.

Proposition 6.4. In $\mathcal{L}_{H \mathbb{F}_{p}}$ there are localizing subcategories that are not Bousfield classes.

Proof. The following counterexample was suggested to us by Mark Hovey. The Bousfield lattice of $\mathcal{L}_{H \mathbb{F}_{p}}$ has only two elements: $\langle 0\rangle=\mathcal{L}_{H \mathbb{F}_{p}}$ and $\mathbb{1}=\{0\}$. It suffices to find a proper nonzero localizing subcategory in $\mathcal{L}_{H \mathbb{F}_{p}}$.

Consider the Moore spectrum $M(p)$, defined by the triangle $S^{0} \stackrel{p}{\rightarrow} S^{0} \rightarrow M(p)$; this spectrum is $H \mathbb{F}_{p}$-local. Consider the following full subcategory in $\mathcal{L}_{H \mathbb{F}_{p}}$.

$$
\mathcal{A}=\left\{X \in \mathcal{L}_{H \mathbb{F}_{p}} \mid[X, M(p)]_{n}=0 \text { for all } n \in \mathbb{Z}\right\} .
$$

This is a localizing subcategory, called the cohomological Bousfield class of $M(p)$ and denoted $\left\langle M(p)^{*}\right\rangle$ in [Hovey 1995b]. The spectrum $H \mathbb{F}_{p}$ is a ring object, and hence local with respect to itself. As mentioned in Section 4, it is known that $H \mathbb{F}_{p}$ is dissonant and $M(p)$ is harmonic, so $\left[H \mathbb{F}_{p}, M(p)\right]_{n}=0$ for all $n$, and $H \mathbb{F}_{p} \in \mathcal{A}$. On the other hand, the identity on $M(p)$ is nonzero, so $M(p) \notin \mathcal{A}$. This shows that $\mathcal{A}$ is a localizing subcategory that is not a Bousfield class.

Another example comes from $Z=L_{H \mathbb{F}_{p}}(B P)$. Clearly $Z \notin\left\langle Z^{*}\right\rangle$. But $B P$ is also harmonic, so $\left[H \mathbb{F}_{p}, B P\right]_{n}=0$ and $\left[H \mathbb{F}_{p}, Z\right]_{n}=0$ for all $n$, and $H \mathbb{F}_{p} \in\left\langle Z^{*}\right\rangle$. Since $Z \in\left\langle M(p)^{*}\right\rangle$, we know that $\left\langle M(p)^{*}\right\rangle \neq\left\langle Z^{*}\right\rangle$. 
Both these counterexamples are cohomological Bousfield classes. It would be interesting to find a localizing subcategory in $\mathcal{L}_{H \mathbb{F}_{p}}$ that is not a cohomological Bousfield class, or show there are none. Also, it is not clear what, if anything, the previous proposition might tell us about the original conjecture in $\mathcal{S}$. For example, as localizing subcategories in $\mathcal{S}$, we have that $\left\langle M(p)^{*}\right\rangle=\langle I\rangle$ [Hovey 1995b, 3.3].

6B. The I-local category. Recall that by $I$ we mean the Brown-Comenetz dual of the sphere spectrum. It is a rare example of a nonzero spectrum that squares to zero. Hovey and Palmieri [1999, Lemma 7.8] conjecture that $\langle I\rangle$ is minimal in $\operatorname{BL}(\mathcal{S})$.

Theorem 6.5. On $\mathcal{L}_{I}$, for all $n$ we have that $\mathrm{LTC}_{n}, \mathrm{LTC}_{n}$, and $\mathrm{LTC}_{n}$ all hold.

Proof. By Lemma 7.1(c) of [Hovey and Palmieri 1999], $T(n) \wedge I=0$ for all $n$, so $L T(n)=0$. Since $K(i)$ is a $B P$-module, and $B P \wedge I=0$ by [Hovey and Strickland 1999, Corollary B.11], we also have $K(n) \wedge I=0$ for all $n$. Therefore $\langle L T(n)\rangle=\langle 0\rangle=\langle L K(n)\rangle$ for all $n$, and the rest follows from Theorem 3.12.

Proposition 6.6. The Bousfield lattice of $\mathcal{L}_{I}$ is the two-element lattice $\left\{\langle 0\rangle,\left\langle L_{I} S^{0}\right\rangle\right\}$.

Proof. By [Hovey and Palmieri 1999, 7.1(c)], $\langle I\rangle\left\langle\left\langle H \mathbb{F}_{p}\right\rangle\right.$. Then Proposition 2.6 implies that there is a surjective lattice map from $\operatorname{BL}\left(\mathcal{L}_{H \mathbb{F}_{p}}\right)=\left\{\langle 0\rangle,\left\langle H \mathbb{F}_{p}\right\rangle\right\}$ onto $\operatorname{BL}\left(\mathcal{L}_{I}\right)$. Note that, by Lemma 2.8, we have $\left\langle L_{I} H \mathbb{F}_{p}\right\rangle=\left\langle L_{I} L_{H \mathbb{F}_{p}} S^{0}\right\rangle=\left\langle L_{I} S^{0}\right\rangle$.

It remains to show that $\left\langle L_{I} S^{0}\right\rangle \neq\langle 0\rangle$. But any $X$ in $\mathcal{S}$ with $X \wedge I \neq 0$ in $\mathcal{S}$ will have $L_{I} X \neq 0$ and $L_{I} X \notin\left\langle L_{I} S^{0}\right\rangle$ in $\operatorname{BL}\left(\mathcal{L}_{I}\right)$; this is due to the fact that $L_{I} X \wedge_{\mathcal{L}_{I}} L_{I} S^{0}=L_{I}\left(L_{I} X \wedge_{\mathcal{S}} L_{I} S^{0}\right)=L_{I}\left(X \wedge_{\mathcal{S}} S^{0}\right)=L_{I}(X)$. For example, $F(n) \wedge I \neq 0$ for all $n$ [Hovey and Palmieri 1999, 7.1(e)].

Corollary 6.7. In $\mathcal{L}_{I}$, the GSC fails but the SDGSC holds.

Proof. Corollary B.13 of [Hovey and Strickland 1999] also shows that $\mathcal{L}_{I}$ has no nonzero compacts, so the proof is the same as for $\mathcal{L}_{H \mathbb{F}_{p}}$.

Hovey states the Dichotomy Conjecture in [1995a, Conjecture 3.10]: In $\mathcal{S}$, every spectrum has either a finite local or a finite acyclic. In [Hovey and Palmieri 1999] the authors discuss several equivalent formulations and some implications. We briefly point out a relationship between this conjecture and Proposition 6.6.

Proposition 6.8. If the Dichotomy Conjecture holds, then the cardinality of $\operatorname{BL}\left(\mathcal{L}_{I}\right)$ is at most two.

Proof. Lemma 7.8 of [Hovey and Palmieri 1999] shows that if the Dichotomy Conjecture holds, then $\langle I\rangle$ is minimal among nonzero classes in $\operatorname{BL}(\mathcal{S})$. This is the case if and only if $a\langle I\rangle$ is maximal among non-top classes in $\operatorname{BL}(\mathcal{S})$, where $a(-)$ denotes the complementation operation first studied by Bousfield [1979b]. Let $a\langle I\rangle \uparrow$ denote the sublattice $\{\langle X\rangle \mid\langle X\rangle \geq a\langle I\rangle\} \subseteq \mathrm{BL}(\mathcal{S})$. In [Wolcott 2014, Proposition 3.2] we show that there is a surjective lattice map from $a\langle I\rangle \uparrow$ onto $\operatorname{BL}\left(\mathcal{L}_{I}\right)$. Thus, if the 
Dichotomy Conjecture holds, $a\langle I\rangle \uparrow$ has cardinality two and $\operatorname{BL}\left(\mathcal{L}_{I}\right)$ has cardinality at most two.

As for classifying localizing subcategories of $\mathcal{L}_{I}$, or at least perhaps finding a proper nonzero localizing subcategory, we must get around the fact that so many spectra are $I$-acyclic. We know that $L F(n) \neq 0$ for all $n$, however $\operatorname{loc}(L F(n))$ is the acyclics of $l_{n-1}^{f}: \mathcal{L}_{I} \rightarrow \mathcal{L}_{I}$ and Theorem 6.5 shows that $l_{n}^{f}=0$ for all $n$. Thus $\operatorname{loc}(L F(n))=\operatorname{loc}\left(L S^{0}\right)$ in $\mathcal{L}_{I}$ for each $n$.

\section{C. The BP-local category.}

Theorem 6.9. On $\mathcal{L}_{B P}$, for all $n$ we have that $\mathrm{LTC}_{n}, \mathrm{LTC}_{n}$, and $\mathrm{LTC}_{n}$ all hold. Proof. We will show that $\mathrm{LTC}_{n}$ holds for all $n$, and the rest follows from Theorem 3.12. Since each $K(i)$ is a $B P$-module spectrum, $\langle K(i)\rangle \leq\langle B P\rangle$, and since $K(i)$ is local with respect to itself this implies that $K(i)$ is $B P$-local. Furthermore, this implies $\langle E(n)\rangle \leq\langle B P\rangle$, so from Lemma 2.8 $L_{n}=L_{n} L=L L_{n}$ as functors on $\mathcal{S}$.

We claim that $L_{n}: \mathcal{L}_{B P} \rightarrow \mathcal{L}_{B P}$, taking $L Y \mapsto L_{n} L Y=L_{n} Y$, is a smashing localization functor on $\mathcal{L}_{B P}$. We have $L_{n}(L Y)=L\left(L_{n} Y\right)=L\left(L_{n} S^{0} \wedge_{\mathcal{S}} Y\right)=$ $L\left(L L_{n} S^{0} \wedge_{\mathcal{S}} L Y\right)=L\left(L_{n} L S^{0} \wedge_{\mathcal{S}} L Y\right)=\left(L_{n} L S^{0}\right) \wedge_{\mathcal{L}_{B P}}(L Y)$. This shows that on $\mathcal{L}_{B P}$ the localization functor $L_{n}$ is also given by smashing with the localization of the unit, $L_{n} L S^{0}$, and thus is smashing.

We know that $L_{n}$ and $l_{n}$ are isomorphic since both are localization functors on $\mathcal{L}_{B P}$ that annihilate $\langle K(0) \vee \cdots \vee K(n)\rangle=\langle L K(0) \vee \cdots \vee L K(n)\rangle$.

On $\mathcal{S}$, the natural map $L_{n}^{f} X \rightarrow L_{n} X$ is a $B P$-equivalence [Ravenel 1993, Theorem 2.7(iii)]. This means that $L L_{n}^{f} X=L L_{n} X$ for all objects $X$ in $\mathcal{S}$, in particular for all $B P$-local objects. Therefore $L L_{n}^{f}=L_{n}=l_{n}$ is a smashing localization functor on $\mathcal{L}_{B P}$. The acyclics are $\left\langle L L_{n}^{f}\left(L S^{0}\right)\right\rangle=\left\langle L L_{n}^{f} S^{0}\right\rangle=\langle L T(0) \vee \cdots \vee L T(n)\rangle$. These are the same acyclics as for $l_{n}^{f}$, and so we conclude that $l_{n}^{f}$ and $l_{n}$ are isomorphic, and the natural map $l_{n}^{f} X \rightarrow l_{n} X$ is an isomorphism.

Proposition 6.10. The GSC fails in $\mathcal{L}_{B P}$.

Proof. The proof of the last theorem showed that $L_{n}: \mathcal{L}_{B P} \rightarrow \mathcal{L}_{B P}$ is a (different) smashing localization for each $n$. However, by [Hovey and Strickland 1999, Corollary B.13] the category $\mathcal{L}_{B P}$ has no nonzero compact objects.

Note that the SDGSC could still hold, since all the smashing localizations we have identified on $\mathcal{L}_{B P}$ are of the form $L_{n}=l_{n}=l_{n}^{f}$, and so are generated by strongly dualizable objects. The question of finding any other smashing localizations on $\mathcal{L}_{B P}$ is probably at least as hard as doing so on $\mathcal{S}$, in light of Proposition 3.16.

All of $\langle E(n)\rangle,\langle K(n)\rangle,\left\langle H \mathbb{F}_{p}\right\rangle$, and $\langle I\rangle$ are "small" in $\operatorname{BL}(\mathcal{S})$, so by Lemma 2.8 it is not surprising that the Bousfield lattices of their localized categories are not very large; this is not true of $\langle B P\rangle$ in $\operatorname{BL}(\mathcal{S})$. We have the following bounds on the Bousfield lattice of the local category. 
Proposition 6.11. The Bousfield lattice of $\mathcal{L}_{B P}$ has $2^{\aleph_{0}} \leq\left|\operatorname{BL}\left(\mathcal{L}_{B P}\right)\right| \leq 2^{2^{\aleph_{0}}}$.

Proof. The second inequality is Corollary 2.7. Since $\langle K(i)\rangle \leq\langle B P\rangle$ for all $i$, we have $\langle Q\rangle=\left\langle\bigvee_{i \geq 0} K(i)\right\rangle \leq\langle B P\rangle$, and so by Propositions 2.6 and 4.2 we have $\left|\mathrm{BL}\left(\mathcal{L}_{B P}\right)\right| \geq|\mathrm{BL}(\mathcal{H})|=2^{\aleph_{0}}$.

6D. The $\boldsymbol{F}(\boldsymbol{n})$-local category. We conclude with a discussion of the $F(n)$-local category.

Any smashing localization $L: \mathcal{S} \rightarrow \mathcal{S}$ gives a splitting of the Bousfield lattice

$$
\mathrm{BL}(\mathcal{S}) \stackrel{\sim}{\longrightarrow} \mathrm{BL}\left(\mathcal{L}_{L S^{0}}\right) \times \mathrm{BL}\left(\mathcal{L}_{C S^{0}}\right),
$$

where $\langle X\rangle \mapsto\left(\left\langle X \wedge L S^{0}\right\rangle,\left\langle X \wedge C S^{0}\right\rangle\right)$. See [Iyengar and Krause 2013, Proposition 6.12] or [Wolcott 2014, Theorem 5.14] for more details. Taking $L=L_{n}^{f}: \mathcal{S} \rightarrow \mathcal{S}$, we have $\left\langle L S^{0}\right\rangle=\langle T(0) \vee \cdots \vee T(n)\rangle$ and $\left\langle C S^{0}\right\rangle=\langle F(n+1)\rangle$. Of course, the relationship between $\mathcal{L}_{T(0) \vee \cdots \vee T(n)}$ and $\mathcal{L}_{E(n)}$ of Section 5A is immediately related to the original $\mathrm{TC}_{n}$ in $\mathcal{S}$. However, this suggests that $\mathcal{L}_{F(n)}$ is worth investigating further.

By Lemma 2.10, in $\operatorname{BL}(\mathcal{S})$ there is a chain

$$
\left\langle S^{0}\right\rangle=\langle F(0)\rangle \geq\langle F(1)\rangle \geq\langle F(2)\rangle \geq \cdots,
$$

and by Lemma 2.8 this gives a chain of lattice surjections

$$
\mathrm{BL}(\mathcal{S})=\mathrm{BL}\left(\mathcal{L}_{F(0)}\right) \rightarrow \mathrm{BL}\left(\mathcal{L}_{F(1)}\right) \rightarrow \mathrm{BL}\left(\mathcal{L}_{F(2)}\right) \rightarrow \cdots .
$$

From the above observations, we expect $\operatorname{BL}\left(\mathcal{L}_{F(n)}\right)$ to be about as complicated as $\operatorname{BL}(\mathcal{S})$. For example, $F(n) \wedge I \neq 0$ for all $n$, and so $L_{F(n)} I$ is a square-zero object in $\mathcal{L}_{F(n)}$. This means that, unlike in most of the localized categories discussed throughout this paper, we know that $\mathrm{BA}\left(\mathcal{L}_{F(n)}\right) \neq \mathrm{BL}\left(\mathcal{L}_{F(n)}\right)$.

\section{Acknowledgements}

We would like to thank Dan Christensen for extensive discussions and suggestions and Jon Beardsley for Proposition 4.2.

\section{References}

[Angeleri Hügel et al. 2008] L. Angeleri Hügel, J. Šaroch, and J. Trlifaj, "On the telescope conjecture for module categories", J. Pure Appl. Algebra 212:2 (2008), 297-310. MR 2008m:16015 Zbl 1141.16010

[Balmer and Favi 2011] P. Balmer and G. Favi, "Generalized tensor idempotents and the telescope conjecture”, Proc. Lond. Math. Soc. (3) 102:6 (2011), 1161-1185. MR 2012d:18010 Zbl 1220.18009

[Benson et al. 2011a] D. Benson, S. B. Iyengar, and H. Krause, "Stratifying triangulated categories", J. Topol. 4:3 (2011), 641-666. MR 2012m:18016 Zbl 1239.18013

[Benson et al. 2011b] D. J. Benson, S. B. Iyengar, and H. Krause, "Stratifying modular representations of finite groups", Ann. of Math. (2) 174:3 (2011), 1643-1684. MR 2846489 Zbl 1261.20057 
[Bousfield 1979a] A. K. Bousfield, "The Boolean algebra of spectra”, Comment. Math. Helv. 54:3 (1979), 368-377. MR 81a:55015 Zbl 0421.55002

[Bousfield 1979b] A. K. Bousfield, "The localization of spectra with respect to homology", Topology 18:4 (1979), 257-281. MR 80m:55006 Zbl 0417.55007

[Brüning 2007] K. Brüning, "Thick subcategories of the derived category of a hereditary algebra", Homology, Homotopy Appl. 9:2 (2007), 165-176. MR 2009d:18018 Zbl 1142.18008

[Casacuberta et al. 2014] C. Casacuberta, J. J. Gutiérrez, and J. Rosický, "A generalization of Ohkawa's theorem”, Compos. Math. 150:5 (2014), 893-902. MR 3209799 Zbl 1300.55007

[Hopkins and Smith 1998] M. J. Hopkins and J. H. Smith, "Nilpotence and stable homotopy theory, II”, Ann. of Math. (2) 148:1 (1998), 1-49. MR 99h:55009 Zbl 0927.55015

[Hovey 1995a] M. Hovey, "Bousfield localization functors and Hopkins' chromatic splitting conjecture”, pp. 225-250 in The Čech centennial (Boston, MA, 1993), edited by M. Cenkl and H. Miller, Contemp. Math. 181, Amer. Math. Soc., Providence, RI, 1995. MR 96m:55010 Zbl 0830.55004

[Hovey 1995b] M. Hovey, "Cohomological Bousfield classes”, J. Pure Appl. Algebra 103:1 (1995), 45-59. MR 96g:55008 Zbl 0841.55008

[Hovey and Palmieri 1999] M. Hovey and J. H. Palmieri, "The structure of the Bousfield lattice", pp. 175-196 in Homotopy invariant algebraic structures (Baltimore, MD, 1998), edited by J.-P. Meyer et al., Contemp. Math. 239, Amer. Math. Soc., Providence, RI, 1999. MR 2000j:55033 Zbl 0947.55014

[Hovey and Strickland 1999] M. Hovey and N. P. Strickland, Morava K-theories and localisation, vol. 139, Mem. Amer. Math. Soc. 666, Amer. Math. Soc., Providence, RI, 1999. MR 99b:55017 Zbl 0929.55010

[Hovey et al. 1997] M. Hovey, J. H. Palmieri, and N. P. Strickland, Axiomatic stable homotopy theory, vol. 128, Mem. Amer. Math. Soc. 610, Amer. Math. Soc., Providence, RI, 1997. MR 98a:55017 Zbl 0881.55001

[Iyengar and Krause 2013] S. B. Iyengar and H. Krause, "The Bousfield lattice of a triangulated category and stratification”, Math. Z. 273:3-4 (2013), 1215-1241. MR 3030697 Zbl 06149061

[Keller 1994] B. Keller, "A remark on the generalized smashing conjecture", Manuscripta Math. 84:2 (1994), 193-198. MR 95h:18014 Zbl 0826.18004

[Krause 2000] H. Krause, "Smashing subcategories and the telescope conjecture - an algebraic approach”, Invent. Math. 139:1 (2000), 99-133. MR 2000k:55016 Zbl 0937.18013

[Krause 2005] H. Krause, "Cohomological quotients and smashing localizations", Amer. J. Math. 127:6 (2005), 1191-1246. MR 2007d:18018 Zbl 1090.18007

[Krause 2010] H. Krause, "Localization theory for triangulated categories", pp. 161-235 in Triangulated categories, edited by T. Holm et al., London Math. Soc. Lecture Note Ser. 375, Cambridge Univ. Press, 2010. MR 2012e:18026 Zbl 1232.18012

[Krause and Solberg 2003] H. Krause and Ø. Solberg, "Applications of cotorsion pairs", J. London Math. Soc. (2) 68:3 (2003), 631-650. MR 2004k:16028 Zbl 1061.16006

[Krause and Št'ovíček 2010] H. Krause and J. Št'ovíček, "The telescope conjecture for hereditary rings via Ext-orthogonal pairs", Adv. Math. 225:5 (2010), 2341-2364. MR 2011j:16013 Zbl 1242.16007

[Mahowald 1982] M. Mahowald, "The image of $J$ in the EHP sequence", Ann. of Math. (2) 116:1 (1982), 65-112. MR 83i:55019 Zbl 0504.55010

[Mahowald and Sadofsky 1995] M. Mahowald and H. Sadofsky, " $v_{n}$ telescopes and the Adams spectral sequence”, Duke Math. J. 78:1 (1995), 101-129. MR 96h:55006 Zbl 0984.55008 
[Mahowald et al. 2001] M. Mahowald, D. Ravenel, and P. Shick, "The triple loop space approach to the telescope conjecture", pp. 217-284 in Homotopy methods in algebraic topology (Boulder, CO, 1999), edited by J. P. C. Greenlees et al., Contemp. Math. 271, Amer. Math. Soc., Providence, RI, 2001. MR 2002g:55014 Zbl 0984.55009

[Miller 1981] H. R. Miller, "On relations between Adams spectral sequences, with an application to the stable homotopy of a Moore space", J. Pure Appl. Algebra 20:3 (1981), 287-312. MR 82f:55029 Zbl 0459.55012

[Miller 1992] H. Miller, "Finite localizations", Bol. Soc. Mat. Mexicana (2) 37:1-2 (1992), 383-389. MR 96h:55009 Zbl 0852.55015

[Neeman 1992] A. Neeman, "The chromatic tower for $D(R)$ ", Topology 31:3 (1992), 519-532. MR 93h:18018 Zbl 0793.18008

[Ohkawa 1989] T. Ohkawa, "The injective hull of homotopy types with respect to generalized homology functors", Hiroshima Math. J. 19:3 (1989), 631-639. MR 90j:55013 Zbl 0697.55008

[Ravenel 1984] D. C. Ravenel, "Localization with respect to certain periodic homology theories", Amer. J. Math. 106:2 (1984), 351-414. MR 85k:55009 Zbl 0586.55003

[Ravenel 1992] D. C. Ravenel, Nilpotence and periodicity in stable homotopy theory, Annals of Mathematics Studies 128, Princeton University Press, 1992. MR 94b:55015 Zbl 0774.55001

[Ravenel 1993] D. C. Ravenel, "Life after the telescope conjecture", pp. 205-222 in Algebraic $K$-theory and algebraic topology (Lake Louise, AB, 1991), edited by P. G. Goerss and J. F. Jardine, NATO Adv. Sci. Inst. Ser. C Math. Phys. Sci. 407, Kluwer Acad. Publ., Dordrecht, 1993. MR 96i:55016 Zbl 0899.55009

[Stevenson 2014] G. Stevenson, "Derived categories of absolutely flat rings", Homology Homotopy Appl. 16:2 (2014), 45-64. MR 3234500 Zbl 06416954

[Št'ovíček 2010] J. Št'ovíček, “Telescope conjecture, idempotent ideals, and the transfinite radical”, Trans. Amer. Math. Soc. 362:3 (2010), 1475-1489. MR 2010j:18024 Zbl 1197.18005

[Wolcott 2014] F. L. Wolcott, "Bousfield lattices of non-Noetherian rings: Some quotients and products", Homology Homotopy Appl. 16:2 (2014), 205-229. MR 3257574 Zbl 06416962

Received July 14, 2014. Revised January 13, 2015.

F. LUKE WOLCOTT

DEPARTMENT OF MATHEMATICS

LAWRENCE UNIVERSITY

711 E BOLDT WAY

SPC 24

APPLETON, WI 54911

UNITED STATES

luke.wolcott@gmail.com 


\title{
PACIFIC JOURNAL OF MATHEMATICS
}

\author{
msp.org/pjm
}

Founded in 1951 by E. F. Beckenbach (1906-1982) and F. Wolf (1904-1989)

\section{EDITORS}

Don Blasius (Managing Editor)

Department of Mathematics

University of California

Los Angeles, CA 90095-1555

blasius@math.ucla.edu

\author{
Paul Balmer \\ Department of Mathematics \\ University of California \\ Los Angeles, CA 90095-1555 \\ balmer@math.ucla.edu \\ Robert Finn \\ Department of Mathematics \\ Stanford University \\ Stanford, CA 94305-2125 \\ finn@math.stanford.edu \\ Sorin Popa \\ Department of Mathematics \\ University of California \\ Los Angeles, CA 90095-1555 \\ popa@math.ucla.edu
}

\author{
Vyjayanthi Chari \\ Department of Mathematics \\ University of California \\ Riverside, CA 92521-0135 \\ chari@math.ucr.edu \\ Kefeng Liu \\ Department of Mathematics \\ University of California \\ Los Angeles, CA 90095-1555 \\ liu@math.ucla.edu \\ Jie Qing \\ Department of Mathematics \\ University of California \\ Santa Cruz, CA 95064 \\ qing@ cats.ucsc.edu
}

\section{PRODUCTION}

Silvio Levy, Scientific Editor, production@msp.org

\section{SUPPORTING INSTITUTIONS}

ACADEMIA SINICA, TAIPEI

CALIFORNIA INST. OF TECHNOLOGY

INST. DE MATEMÁTICA PURA E APLICADA

KEIO UNIVERSITY

MATH. SCIENCES RESEARCH INSTITUTE

NEW MEXICO STATE UNIV.

OREGON STATE UNIV.

\author{
STANFORD UNIVERSITY \\ UNIV. OF BRITISH COLUMBIA \\ UNIV. OF CALIFORNIA, BERKELEY \\ UNIV. OF CALIFORNIA, DAVIS \\ UNIV. OF CALIFORNIA, LOS ANGELES \\ UNIV. OF CALIFORNIA, RIVERSIDE \\ UNIV. OF CALIFORNIA, SAN DIEGO \\ UNIV. OF CALIF., SANTA BARBARA
}

\author{
Daryl Cooper \\ Department of Mathematics \\ University of California \\ Santa Barbara, CA 93106-3080 \\ cooper@math.ucsb.edu \\ Jiang-Hua Lu \\ Department of Mathematics \\ The University of Hong Kong \\ Pokfulam Rd., Hong Kong \\ jhlu@maths.hku.hk \\ Paul Yang \\ Department of Mathematics \\ Princeton University \\ Princeton NJ 08544-1000 \\ yang@math.princeton.edu
}

These supporting institutions contribute to the cost of publication of this Journal, but they are not owners or publishers and have no responsibility for its contents or policies.

See inside back cover or msp.org/pjm for submission instructions.

The subscription price for 2015 is US \$420/year for the electronic version, and \$570/year for print and electronic.

Subscriptions, requests for back issues and changes of subscribers address should be sent to Pacific Journal of Mathematics, P.O. Box 4163, Berkeley, CA 94704-0163, U.S.A. The Pacific Journal of Mathematics is indexed by Mathematical Reviews, Zentralblatt MATH, PASCAL CNRS Index, Referativnyi Zhurnal, Current Mathematical Publications and Web of Knowledge (Science Citation Index).

The Pacific Journal of Mathematics (ISSN 0030-8730) at the University of California, c/o Department of Mathematics, 798 Evans Hall \#3840, Berkeley, CA 94720-3840, is published twelve times a year. Periodical rate postage paid at Berkeley, CA 94704, and additional mailing offices. POSTMASTER: send address changes to Pacific Journal of Mathematics, P.O. Box 4163, Berkeley, CA 94704-0163.

PJM peer review and production are managed by EditFLOW ${ }^{\circledR}$ from Mathematical Sciences Publishers.

\section{PUBLISHED BY}

\section{mathematical sciences publishers \\ nonprofit scientific publishing}

http://msp.org/

(C) 2015 Mathematical Sciences Publishers 


\section{PACIFIC JOURNAL OF MATHEMATICS}

Volume $276 \quad$ No. $2 \quad$ August 2015

Free evolution on algebras with two states, II

257

MiCHAEL ANSHELEVICH

Systems of parameters and holonomicity of A-hypergeometric systems

281

Christine Berkesch Zamaere, STEPHEN GRIFfeth and EZRA Miller

Complex interpolation and twisted twisted Hilbert spaces

287

FÉlix Cabello SÁnChez, Jesús M. F. Castillo and Nigel J. Kalton

The ramification group filtrations of certain function field extensions

309

JEFFREY A. CASTAÑEDA and QINGQUAN WU

A mean field type flow, II: Existence and convergence

JEAN-BAPTISTE CASTÉRAS

Isometric embedding of negatively curved complete surfaces in Lorentz-Minkowski space

BING-LONG CHEN and LE YIN

The complex Monge-Ampère equation on some compact Hermitian manifolds

JIANCHUN CHU

Topological and physical link theory are distinct

ALEXANDER COWARD and JOEL HASS

The measures of asymmetry for coproducts of convex bodies

Qi GuO, JinFEng GuO and XunLi Su

Regularity and analyticity of solutions in a direction for elliptic equations

YongYANG Jin, DONGSHENG LI and XU-JIA WANG

On the density theorem for the subdifferential of convex functions on Hadamard spaces

Mina Movahedi, Daryoush Behmardi and Seyedehsomayeh

HOSSEINI

$L^{p}$ regularity of weighted Szegó projections on the unit disc

SAMANGi MunASINGHE and Yunus E. ZEYTUnCU

Topology of complete Finsler manifolds admitting convex functions

SORIN V. SABAU and KatSUHIRO SHIOHAMA

Variations of the telescope conjecture and Bousfield lattices for localized categories of spectra

F. LUKE WOLCOTT 